\title{
Fyn phosphorylates AMPK to inhibit AMPK activity and AMP- dependent activation of autophagy
}

\author{
Eijiro Yamada ${ }^{1}$, Shuichi Okada ${ }^{1}$, Claire C. Bastie ${ }^{2}$, Manu Vatish ${ }^{4}$, Yasuyo Nakajima ${ }^{1}$, \\ Ryo Shibusawa $^{1}$, Atsushi Ozawa ${ }^{1}$, Jeffrey E. Pessin ${ }^{3}$ and Masanobu Yamada ${ }^{1}$ \\ ${ }^{1}$ Department of Medicine and Molecular Science, Gunma University Graduate School of Medicine, Maebashi, Japan \\ 2 Division of Biomedical Sciences, Warwick Medical School, Coventry, West Midlands, United Kingdom \\ ${ }^{3}$ Departments of Medicine and Molecular Pharmacology, Albert Einstein College of Medicine, Bronx, New York, United States \\ of America \\ ${ }^{4}$ Nuffield Department of Obstetrics \& Gynaecology, University of Oxford, Oxford, United Kingdom \\ Correspondence to: Eijiro Yamada, email: eijiro.yamada@gunma-u.ac.jp \\ Keywords: Fyn, AMPK, TNFa, Autophagy
}

Received: March 23, $2016 \quad$ Accepted: September 05, 2016

Published: September 08, 2016

\section{ABSTRACT}

We previously demonstrated that proto-oncogene Fyn decreased energy expenditure and increased metabolic phenotypes. Also Fyn decreased autophagymediated muscle mass by directly inhibiting LKB1 and stimulating STAT3 activities, respectively. AMPK, a downstream target of LKB1, was recently identified as a key molecule controlling autophagy. Here we identified that Fyn phosphorylates the a subunit of AMPK on Y436 and inhibits AMPK enzymatic activity without altering the assembly state of the AMPK heterotrimeric complex. As pro-inflammatory mediators are reported modulators of the autophagy processes, treatment with the proinflammatory cytokine TNFa resulted in 1) increased Fyn activity 2) stimulated Fyndependent AMPKa tyrosine phosphorylation and 3) decreased AICAR-dependent AMPK activation. Importantly, TNFa induced inhibition of autophagy was not observed when AMPKa was mutated on Y436. 4) These data demonstrate that Fyn plays an important role in relaying the effects of TNFa on autophagy and apoptosis via phosphorylation and inhibition of AMPK.

\section{INTRODUCTION}

Although autophagy is originally described as an evolutionarily conserved cellular recycling program $[1,2]$, recent studies have demonstrated the role of autophagy in metabolic functions. Indeed autophagy participates in hepatic lipid storage [3], glucose homeostasis [4, 5], adipocyte differentiation [6, 7] and protects $\alpha$ cells against chronic lipid stress [8, 9]. Interestingly, deregulation of these functions is also associated with inflammatory processes, suggesting that inflammatory molecules modulate the autophagy function. In line with this, prolonged exposure of tissues and organs to high concentrations of inflammatory mediators inhibits autophagy response, resulting in severe cell damage and apoptosis $[10,11]$.

The molecular mechanisms underlining the cross talk between autophagy processes, energy metabolism and immune function have been under scrutiny. Recently, molecules traditionally known for their role in metabolism and homeostasis have been identified as elements of the autophagy pathway. Amongst them is the AMP-dependent protein kinase [AMPK], one of the major energy sensors of the cell $[1,12,13]$. AMPK is a highly conserved heterotrimeric complex composed of three subunits $\alpha, \beta$ and $\gamma$ [14-16]. AMP binds to the $\gamma$ subunit and increases the phosphorylation of the catalytic $\alpha$ subunit $\mathrm{T} 172$ residue by upstream kinases such as $\mathrm{Ca}^{2+/}$ calmodulin-dependent protein kinase kinase (CaMKK2) in the brain and Liver Kinase B1 (LKB1) in peripheral organs $[17,18]$, resulting in the activation of AMPK kinase. The relative expression levels, association, and subcellular distribution of these upstream kinases in addition to the expression levels of the various AMPK $\alpha, \beta, \gamma$ subunit isoforms are responsible for the large pleiotropic responses of AMPK under a variety of cellular states [19].

Although the link between AMPK and autophagy is established, upstream regulators of AMPK in the 
context of autophagy response are still largely unknown. Previously, we reported that Fyn knockout (FynKO) mice display increased AMPK activity as a result of Fyndependent LKB1 regulation in peripheral tissues [20, 21]. Another recent publication also demonstrates that Fyn exerts its inhibitory effect on AMPK through both LKB1 and PIKE-A pathways [22]. Fyn is a member of the Src family of proto-oncogene non-receptor tyrosine kinases with multiple functions including the regulation of several inflammatory processes [23, 24]. Two major spliced isoforms, FynB and FynT have been reported [25], differing by their kinase activity (Fyn $\mathrm{T}$ being the most active isoform) and functions [26]. Interestingly, using muscle specific FynB or FynT over-expressing animals we recently demonstrated Fyn-dependent inhibition of autophagy via STAT3 regulation in muscle [27]. Although this pathway accounts for the ability of Fyn to suppress autophagy under basal conditions, other signalling events are likely to be involved under pathological conditions, particularly under metabolic stress, a condition associated with increased inflammatory processes and production of metabolic cytokines such as TNF $\alpha$ [28]. Interestingly, studies show that TNF $\alpha$ inhibits autophagy processes $[10$, 29] and others suggest that TNF $\alpha$ regulates AMPK activity $[28,30-32]$. Yet, it has not been fully examined whether TNF $\alpha$ inhibits autophagy through AMPK.

In this study, we demonstrate that 1) Fyn specifically phosphorylates AMPK $\alpha$ on Y436, resulting in AMPK activity inhibition and thereby suppressing autophagy, 2) TNF $\alpha$ activates Fyn kinase, 3) Fyn deficiency blocks TNF $\alpha$ inhibition of autophagy by preventing AMPK $\alpha$ subunit Y436 phosphorylation and 4) Fyn deficiency protects against TNF $\alpha$ activation of apoptosis.

\section{RESULTS}

\section{AMPK undergoes Fyn-dependent tyrosine phosphorylation}

Having recently identified LKB1 and STAT3 as direct substrates of Fyn in the respective regulation of metabolism [21] and muscle autophagy [27], we wished to identify other potential Fyn targets. Skeletal muscle extracts from Fyn over-expressing and FynKO mice were subjected to mass spectrometry analysis [27]. Along with the increased STAT3 Y705 phosphorylation in Fyn over-expressing mice, we also found a marked increase in phosphorylation on Y436 of the AMPK $\alpha$ subunit (Supplementary Figure 1A, 1B), suggesting that Y436 could be a specific site for Fyn.

To confirm Fyn-dependent tyrosine phosphorylation of AMPK, gastrocnemius muscle tissue extracts were prepared from Fyn knockout (FynKO), wild type (WT), skeletal muscle-specific over expressing FynB and FynT mice. The extracts were immunoblotted for AMPK $\alpha$ subunit and GADPH as a loading control (Figure 1A). Immunoprecipitation of these extracts with the $4 \mathrm{G} 10$ phospho-tyrosine specific antibody demonstrated a low level of AMPK tyrosine phosphorylation in the FynKO mice compared to control WT mice (Figure 1B). In contrast, skeletal muscle extracts from the FynB and FynT overexpressing mice displayed an approximate 2-fold increase in AMPK $\alpha$ subunit tyrosine phosphorylation compared to wild type mice. FynT is the most active isoform of Fyn, yet AMPK $\alpha$ phosphorylation levels in FynT mice were similar to those seen in FynB mice. However, we found that total AMPK $\alpha$ expression (Figure 1A) in FynT muscle was decreased compared to WT, FynKO or FynB mice, likely due to the fact that FynT mice display a strong sarcopenic phenotype [27] known to decrease AMPK $\alpha$ expression $[28,33]$. Therefore, when normalized to total AMPK $\alpha$, protein levels, tyrosine phosphorylation of AMPK $\alpha$ in FynT mice was further increased when compared to FynB mice (Figure 1C).

To determine if Fyn phosphorylates the AMPK $\alpha$ in cell culture conditions, we co-expressed an epitope tagged AMPK $\alpha 2$ (AMPK $\alpha 2$-Flag) cDNA with or without an epitope tagged constitutively active Fyn (V5-Fyn-CA) cDNA in NIH-3T3 cells (Figure 2A). Expression of V5Fyn-CA resulted in the robust tyrosine phosphorylation levels of AMPK $\alpha 2$-Flag (Figure 2B). We recently reported that Fyn directly phosphorylates LKB1 to modulate AMPK activity [21]. To evaluate whether AMPK tyrosine phosphorylation is also dependent on LKB1, we coexpressed LKB1 in HEK293T cells. LKB1 had no effect on V5-Fyn-CA or AMPKa2-Flag expression. Importantly, tyrosine phosphorylation of AMPKa2-Flag in presence of Fyn was unaffected by LKB1 co-expression (Figure 2C, 2D, 2E). These data demonstrate that Fyn-dependent tyrosine phosphorylation of AMPK $\alpha$ is independent of LKB1.

To demonstrate that AMPK $\alpha$ is a direct Fyn substrate, we incubated human purified AMPK $\alpha 2$ protein with purified Fyn-CA (Figure 2F). In the presence of ATP, Fyn was observed to robustly phosphorylate the AMPK $\alpha 2$ subunit. The specificity for Y436 was confirmed by expression of the Y436F AMPK $\alpha$ mutant (Y436FAMPKa2-Flag) with V5-FynCA in HeLa cells. As readily apparent in Figure $2 \mathrm{G}$ and $2 \mathrm{H}$, the ability of V5-FynCA to tyrosine phosphorylate the Y436F AMPK $\alpha 2$-Flag mutant was reduced compared to WT AMPKa2-Flag, demonstrating that Fyn specifically phosphorylates AMPK $\alpha 2$ on Y436.

\section{Tyrosine phosphorylation of the AMPK $\alpha 2$ subunit residue Y436 inhibits AMPK activity}

To evaluate whether tyrosine phosphorylation on Y436 has an effect on AMPK activity, we silenced the endogenous AMPK $\alpha 1$ and $\alpha 2$ subunits in HEK293T 
A

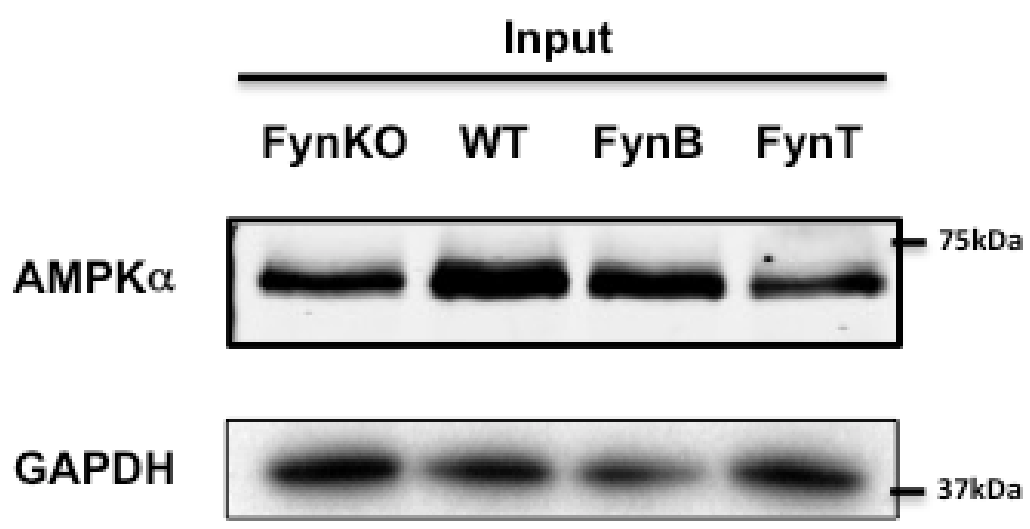

B

IP: P-Tyr

\section{FynKo WT FynB FynT}

AMPK $\alpha \longdiv { \text { nas } }$

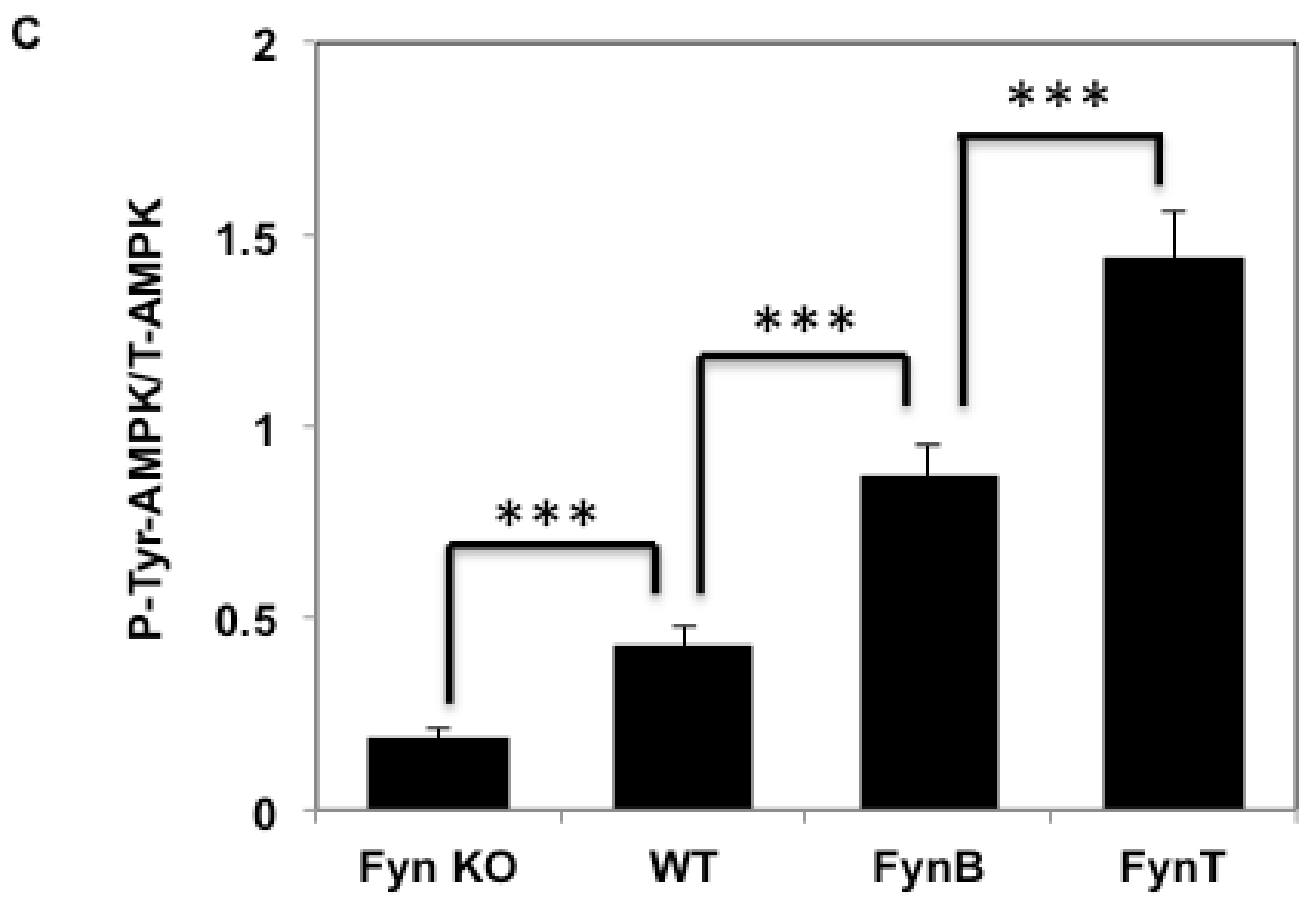

Figure 1: Fyn phosphorylates AMPKa in skeletal muscle. A. Whole cell lysates from gastrocnemius muscle of either FynKO mice, WT, HSA-FynB (FynB) or HSA-FynT (FynT) mice were homogenized and immnoblots were performed by AMPK $\alpha$ mAb and GAPDH B. Gastrocnemius muscle of either FynKO mice, WT, HSA-FynB (FynB) or HSA-FynT (FynT) mice were homogenized and immunoprecipitated with $4 \mathrm{G} 10 \mathrm{mAb}$ followed by immunoblotting with AMPK mAb. Blots are representative of 3 independent experiments. C. Signal quantification of tyrosine-phosphorylated AMPK $\alpha$ levels from (A) and (B). Statistical examination between each set was performed. $* * * P<0.005$. 
A

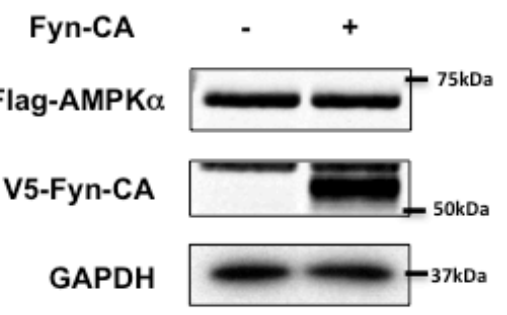

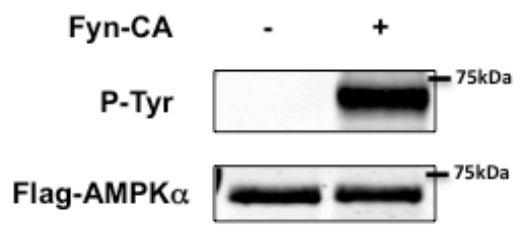

C
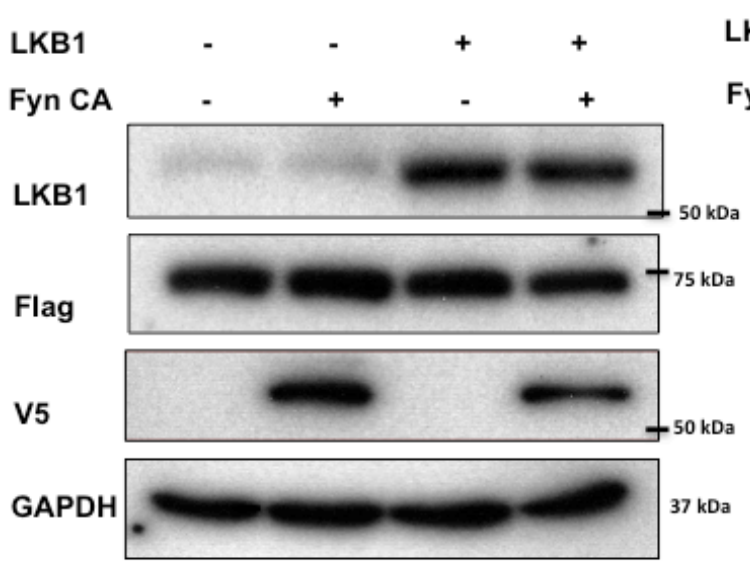

F

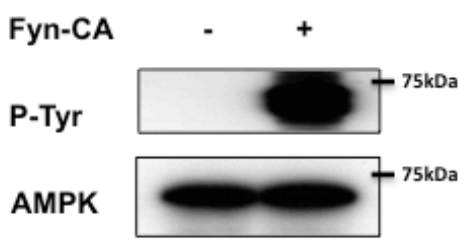

D

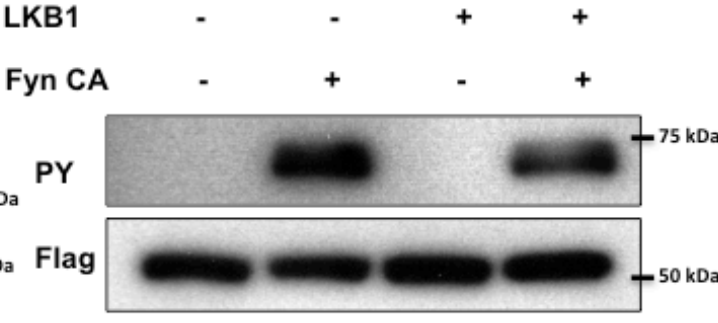

E
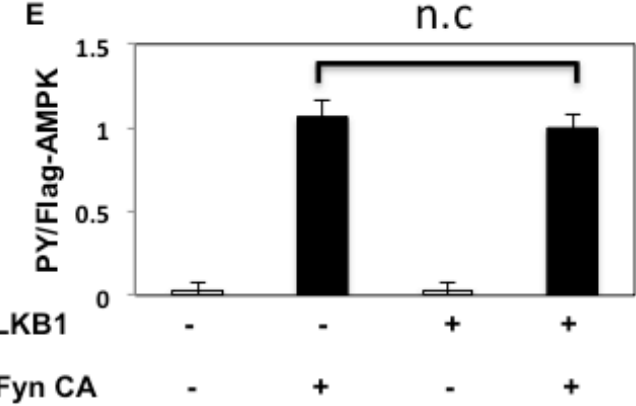

G

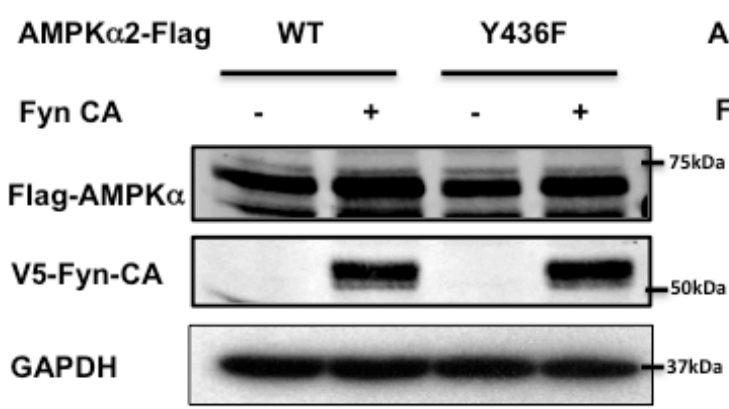

H

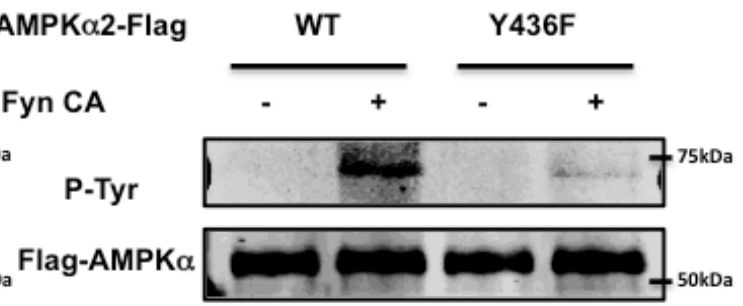

Figure 2: Fyn phosphorylates AMPK on tyrosine 436. A., B. NIH3T3 cells were co-transfected with $3 \mathrm{myc}-\mathrm{AMPK} \alpha 2$-Flag and Fyn-CA-V5. $48 \mathrm{~h}$ later, cells extracts were prepared and immunoprecipitated with a Flag antibody. Immunoblots of lysates (A) and immunoprecipitation samples (B) were performed with the indicated antibodies. Blots are representative of 3 independent experiments. C., D. Forty-eight hours after co-transfection with 3myc-AMPK $\alpha 2$-Flag, Fyn-CA-V5 and pcDNA3-LKB1, HEK293T cells extracts were prepared and immunoprecipitation was performed using a Flag antibody. Immunoblots of lysates (C) and immunoprecipitation samples (D) were performed with the indicated antibodies. These are representative immunoblots independently performed 3 times. E. Signal quantification of tyrosine-phosphorylated AMPK $\alpha$ levels from (D). Statistical examination between each set was performed. F. Purified Human PRKAA2 (100 ng) and either mock or Flag-Fyn-CA (380 ng) were incubated with the kinase buffer and ATP at $30^{\circ} \mathrm{C}$ for 30 min. Samples were boiled and separated onto a $8 \%$ SDS-PAGE gel, followed by immunoblotting with the indicated antibodies. G., H. HeLa cells were co-transfected by either 3myc-AMPK $\alpha 2$ WT or Y436F (YF) with Fyn-CA-V5. Immunoprecipitation was performed using a Flag antibody. Immunoblots of lysates $(\mathrm{G})$ and immunoprecipitation samples $(\mathrm{H})$ were performed with the indicated antibodies. Images are representative of 4 independent experiments. 
cells using specific human siRNA. As shown in Figure $3 \mathrm{~A}$, siRNA-mediated knockdown of AMPK $\alpha$ greatly reduced endogenous AMPK $\alpha$ protein expression levels. The knockdown cells were then transfected with an empty vector (pcDNA3), rat GST-AMPK $\alpha 1-\mathrm{WT}$ (WT) or the GST-AMPK 1 1-Y430F mutant (the equivalent to human Y436 of AMPK $\alpha 2$ subunit (Y436F)). We selected these rat constructs as they are not affected by human AMPK $\alpha$ siRNA and therefore continue to be expressed in HEK293T cells transfected by human AMPK $\alpha$ siRNA. Phosphorylation of acetyl-CoA carboxylase (ACC) on S79 (pS79-ACC), the specific AMPK site on ACC [34, 35] was used as a read-out for AMPK activity. As apparent in Figure $3 \mathrm{~B}$ and $3 \mathrm{C}$, siRNA mediated knockdown of endogenous AMPK reduced the basal level of pS79ACC. Re-expression of rat GST-AMPK $\alpha 1-\mathrm{WT}$ resulted in a relatively small but significant increase in pS79ACC whereas re-expression of rat GST-AMPK $\alpha 1-Y 430 F$ increased ACC phosphorylation to a greater extent. The relative weak effect of rat GST-AMPK $\alpha 1-\mathrm{WT}$ to reconstitute AMPK dependent ACC phosphorylation was likely due to either the use of rat GST-AMPK $\alpha 1$ fusion protein or it might be that the rat protein does not fully compliment the human $\alpha 1$ subunit in HEK293T cells. Nevertheless, these data demonstrate that the Y436F AMPK $\alpha$ mutant displays significantly more AMPK activity than WT-AMPK $\alpha$.

Recently, studies have demonstrated that AMPK kinase activity is regulated by modification of the AMPK $\alpha, \beta, \gamma$ subunit trimeric complex assembly state $[14,15$, 17]. To determine whether Fyn suppresses AMPK activity through dissociation of the $\alpha, \beta, \gamma$ subunit interactions, we performed a series of co-immunoprecipitation experiments (Figure 3D, 3E and supplementary Figure $2 \mathrm{~A}$ and $2 \mathrm{~B}$ ). Co-immunoprecipitation analyses revealed that phosphorylation of AMPK Y436 had no effect on the assembly status of the AMPK trimeric complex, indicating that Fyn-dependent phosphorylation and inhibition of AMPK activity does not result from AMPK subunit dissociation.

\section{Prolonged TNF $\alpha$ treatment diminishes AICAR- dependent AMPK activation through Fyn}

Although a number of studies have shown that the pro-inflammatory cytokine $\mathrm{TNF} \alpha$ regulates $\mathrm{AMPK}$ activity $[10,29]$, the molecular mechanisms underlying this regulation are still unclear [10, 29, 31, 32]. To test the hypothesis that TNF $\alpha$ might regulate AMPK through Fyn-dependent phosphorylation, we first examined acute and prolonged effects of TNF $\alpha$ incubation on AMPK T172 phosphorylation levels.

Short term (12 h) of TNF $\alpha$ treatment enhanced both basal and AICAR-stimulated T172 AMPK $\alpha$ phosphorylation levels (Figure 4A and 4B). In contrast, while prolonged incubation with TNFa (24-36 h) still enhanced T172 phosphorylation levels in basal condition, additional AICAR-dependent phosphorylation was abolished. Similarly, AICAR-dependent ACC phosphorylation was also further enhanced by $\mathrm{TNF} \alpha$ treatment at $12 \mathrm{~h}$ but not at 24 or $36 \mathrm{~h}$ (Figure 4A and 4C). TNF $\alpha$ treatment also enhanced Fyn kinase activity in a cell extract assay (Figure 5A), without any significant change in Fyn protein levels (Figure 5B). Moreover this was associated with increased endogenous AMPK $\alpha$ subunit tyrosine phosphorylation (Figure 5C and 5D).

To further investigate the role of Fyn in relaying the effects of TNF $\alpha$ on AMPK $\alpha$ phosphorylation, we next silenced Fyn protein expression by siRNA in HEK293T cells. As shown in Figure 6, AICAR increased AMPK $\alpha$ phosphorylation (Figure 6A and 6B) and subsequent ACC phosphorylation (Figure 6A and 6C) in non-target siRNA transfected HEK293T cells. Prolonged TNF $\alpha$ treatment increased the basal state of AMPK $\alpha$ phosphorylation but not that of ACC, suggesting that despite the increase of T172 phosphorylation, AMPK activity was not optimal. Additionally, neither AMPK $\alpha$ nor ACC phosphorylation levels were increased following AICAR stimulation, demonstrating that chronic TNF $\alpha$ blunts the effect of AICAR. However, AMPK and ACC phosphorylation levels were restored and increased in Fyn knockdown cells, suggesting that lack of Fyn the HEK293T cells prevented TNF $\alpha$ inhibition of AICAR-stimulated AMPK $\alpha$ T172 phosphorylation and activation.

\section{Fyn regulates autophagy through AMPK phosphorylation to regulate apoptosis}

As AMPK plays an important regulatory function in the control of autophagy $[1,36]$, we next examined the role of Fyn-dependent AMPK regulation in this process. For this, HEK293T cells in which endogenous AMPK $\alpha 1$ and $\alpha 2$ subunits were silenced (as in Figure 3 ) were transfected with the empty vector (pcDNA3), GST-AMPK $\alpha$-WT (WT) or the GST-AMPK $\alpha 1-Y 430 F$ mutant (the equivalent to human Y436 of AMPK $\alpha 2$ subunit (Y436F)). ULK1 phosphorylation on the AMPK activating site was then quantified $[12,13]$. GST-AMPKWT expression increased ULK1 phosphorylation and expression of the rat GST-AMPK-Y430F mutant resulted in a further increase (Figure 7A). We next examined the effect of Fyn knockdown on ULK1 phosphorylation levels in basal, AICAR-stimulated and prolonged TNF $\alpha$ stimulated cells (Figure 7B and 7C). AICAR significantly increased ULK1 phosphorylation indicating that autophagy was up regulated, and TNF $\alpha$ blunted this effect. Importantly siRNA mediated knockdown of Fyn prevented the chronic TNFa inhibition of AICAR-stimulated ULK1 phosphorylation (Figure 7B and 7C). In line with this, AICAR increased the ratio of LC3-II/LC3-I and 

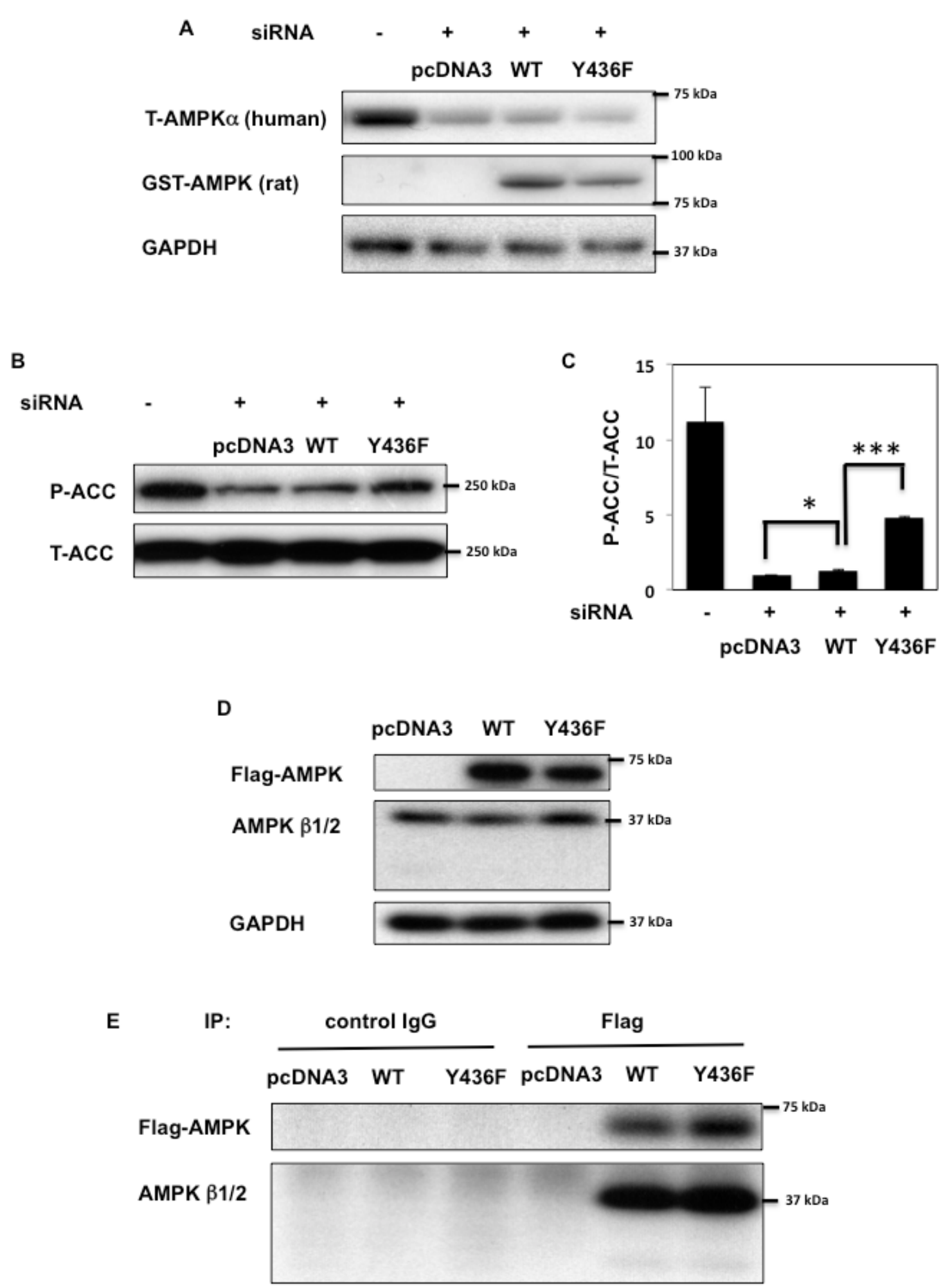

Figure 3: AMPK activity is decreased by tyrosine phosphorylation. A. HEK293T cells were co-transfected with human siAMPK $\alpha 1$ and $\alpha 2$ subunits and either empty vector (pcDNA3), GST-rat AMPK $\alpha$ WT (WT) or Y430F mutant (equivalent to human Y436F (Y436F)) that are not affected by human AMPKa siRNA. $72 \mathrm{~h}$ after transfection immunoblots were performed with the indicated antibodies. B., C. S79 phosphorylation of ACC and total ACC levels in samples from (A) were assessed (B) and signal quantification of the expression levels of S79 phosphorylation of ACC normalized with total ACC were performed (C). Statistical examination between each sets were performed $* p<0.05, * * * p<0.005$. D., E. HEK293T cells were transfected by either 3myc-AMPK $\alpha 2$ WT or Y436F-Flag (Y436F). Co-immunoprecipitation was performed by either control IgG or AMPK $\beta 1 / \beta 2$ polyclonal antibody. Immunoblots of lysates (D) and immunoprecipitation samples (E) were performed with the indicated antibodies. Images are representative of 3 independent experiments. 
A

TNF $\alpha, 12$ h TNF $\alpha, 24$ h

$\mathrm{TNF} \alpha, 36 \mathrm{~h}$
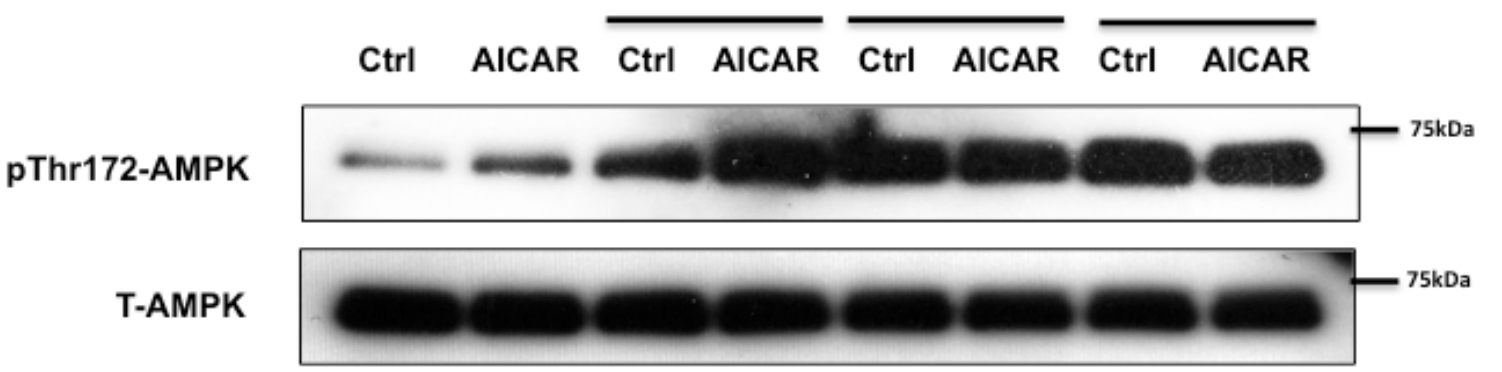

P-ACC

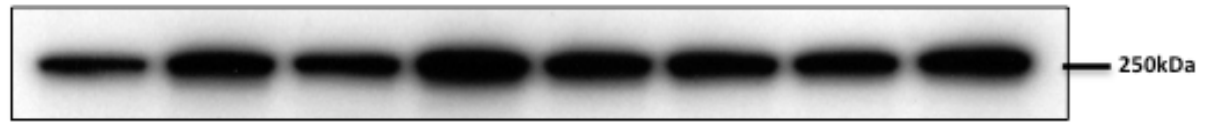

T-ACC

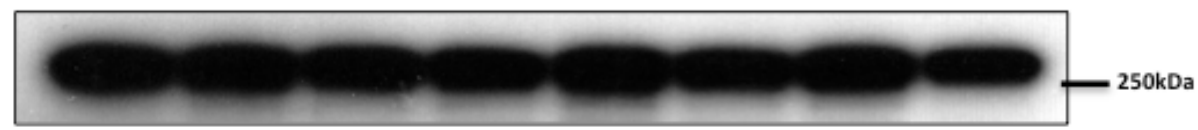

Fyn

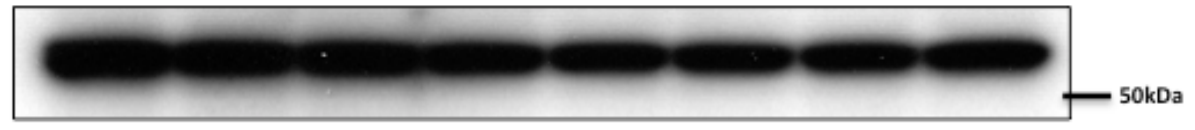

Tubulin $\alpha$

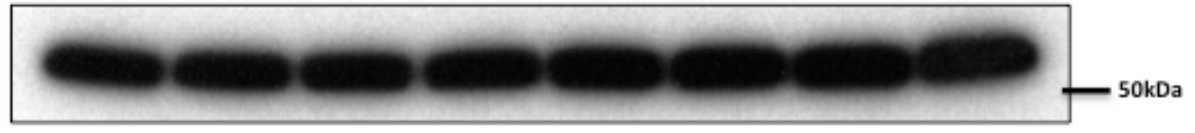

B

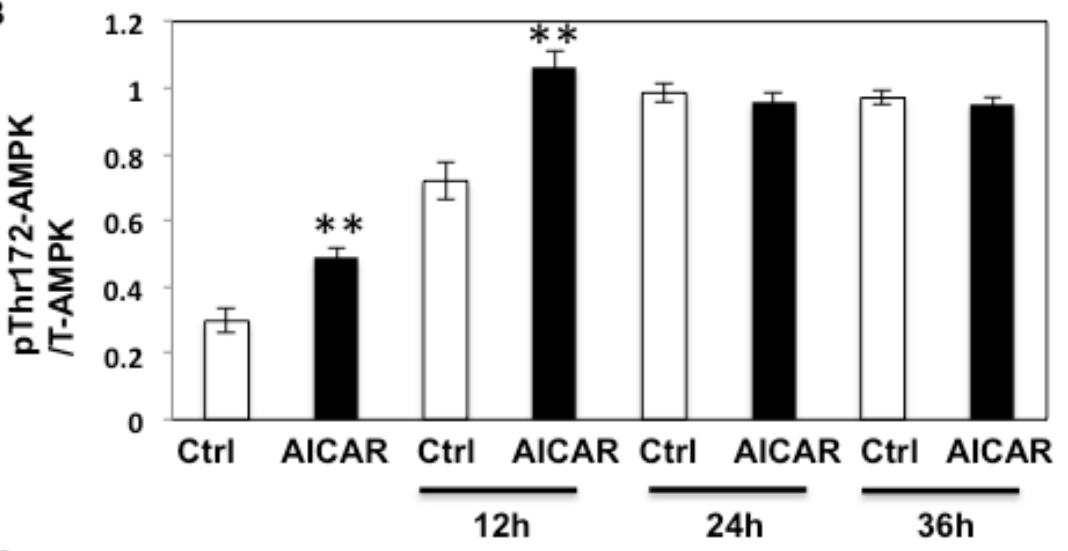

C

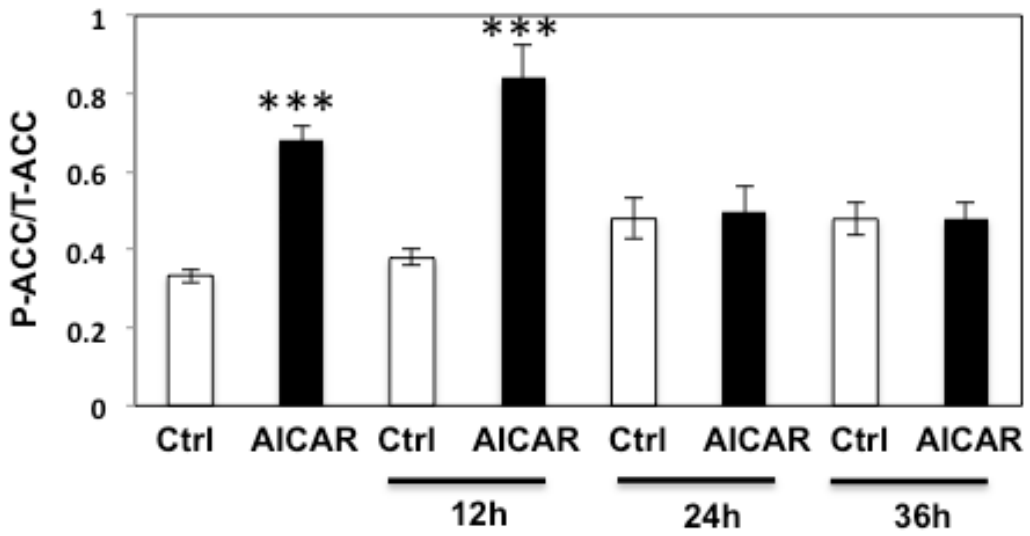

Figure 4: Prolonged TNF $\alpha$ stimulation suppresses AICAR dependent AMPK activation. A. HEK293T cells were incubated with TNF $\alpha(10 \mathrm{ng} / \mathrm{ml})$ for 12,24 or $36 \mathrm{~h}$ and then stimulated with $2 \mathrm{mM}$ AICAR for $10 \mathrm{~min}$. Immunoblots were performed with the indicated antibodies. These are representative images from 3 independent experiments. B., C. Signal quantifications of expression levels of phospho- T172 AMPK $\alpha$ corrected by total AMPK $\alpha$ (B) and levels of phospho- S79 of ACC to total ACC (C). The data are presented as mean \pm s.e.m. Statistical examination between control and AICAR stimulated cells in each sets were performed. ${ }^{* *} p<0.01,{ }^{* * *} p<0.005$. 
A

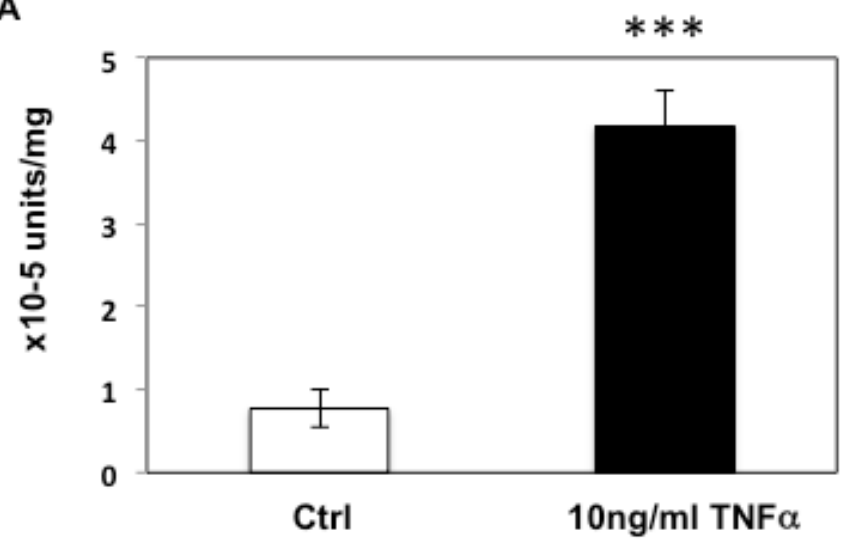

B

Ctrl TNF $\alpha$

Fyn

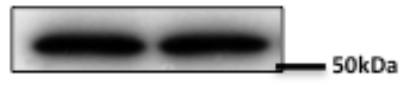

GAPDH

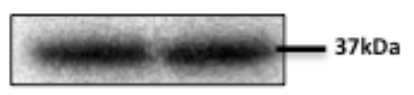

C

Ctrl TNF $\alpha$

P-Tyr

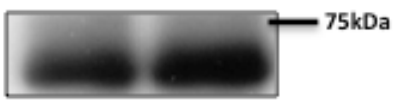

T-AMPK

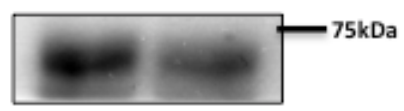

GAPDH

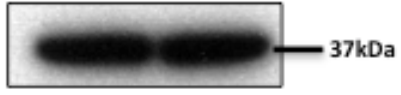

D

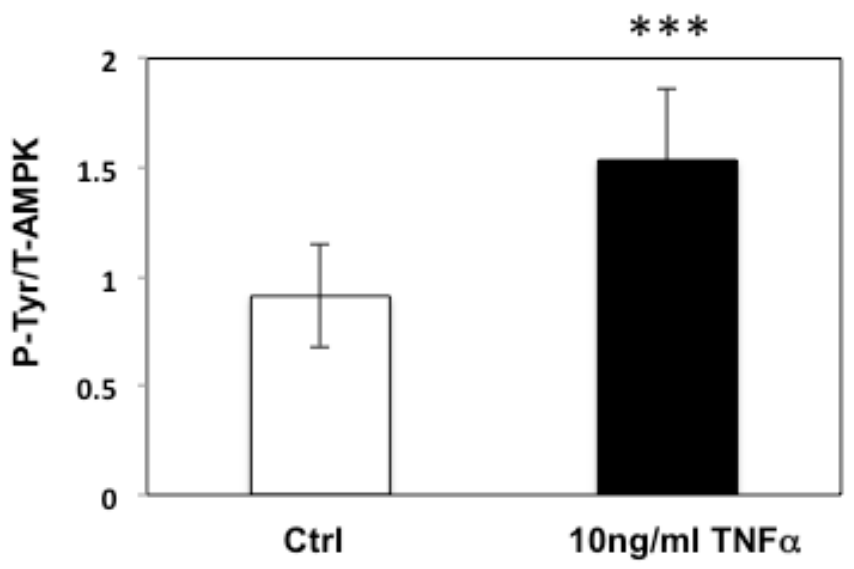

Figure 5: Prolonged TNF $\alpha$ stimulation up-regulates Fyn activity in HEK293T cells. A. Fyn kinase activity in HEK293T cells treated or not with TNF $\alpha(10 \mathrm{ng} / \mathrm{ml})$ for $36 \mathrm{~h}$. These are representative images from 5 independent experiments $* * * p<0.005$. B. Fyn expression in HEK293T cells treated or not with TNF $\alpha$ for 36 h. C. After a 36-h incubation with 10ng/ml TNF $\alpha$, HEK293T cells were treated or not with TNF $\alpha$ for $36 \mathrm{~h}$ and homogenized. Immunoprecipitation was performed with total AMPK $\alpha$ antibody followed by immunoblotting with the 4G10 and total AMPK $\alpha$ antibody. These are representative images from 4 independent experiments. D. Signal quantification (Y436 normalized to total AMPK $\alpha$ ) from (C). $\left({ }^{* * *} P<0.005\right)$ 


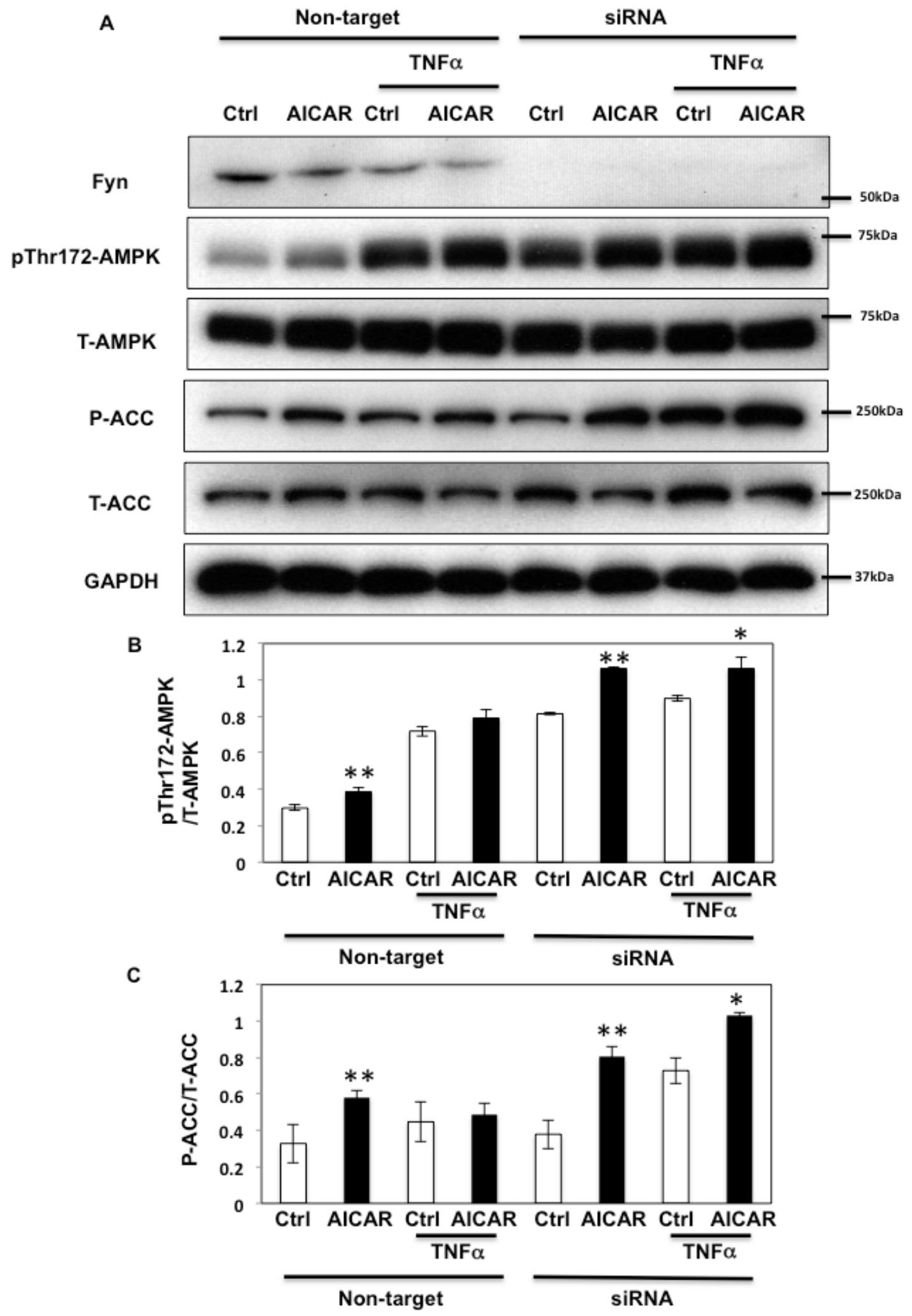

Figure 6: TNF $\alpha$ regulates AICAR-dependent AMPK activation via Fyn kinase. A. HEK293T cells were transfected with Fyn siRNAs. Cells were treated with TNF $\alpha(10 \mathrm{ng} / \mathrm{ml})$ for $36 \mathrm{~h}$ with or without $2 \mathrm{mM}$ AICAR for $10 \mathrm{~min}$. Immunoblots were performed with the indicated antibodies. These are representative images from 3 independent experiments. B., C. Signal quantifications of expression levels of phospho- T172 AMPK $\alpha$ normalized to total AMPK $\alpha$ (B) and levels of S79 phosphorylation of ACC normalized to total ACC (C). The data are presented as mean \pm SE. Statistical examination between control and AICAR stimulated cells in each sets were performed. $* P<$ $0.05, * * P<0.01$. 
decreased expression of $\mathrm{p} 62$, consistent with an increase in autophagic activity (Figure 8A, 8B and 8C). While this was blunted when cells were treated with TNF $\alpha$, siRNAmediated knockdown of Fyn prevented this chronic TNFo inhibition of AICAR-stimulated autophagy activation. AICAR is an adenosine analog that directly modulates AMPK activity, and these results suggested the direct TNF $\alpha$-Fyn regulation of the AMPK-autophagy axis. To confirm that Fyn mediates autophagy via AMPK we next took advantage of metformin, a known AMPK activator that is also an autophagy inducer. As shown Figure $8 \mathrm{D}$, $8 \mathrm{E}$ and $8 \mathrm{~F}$, metformin increased the ratio of LC3-II/LC3-I and decreased p62 in the basal state, which is consistent with an increase in autophagic activity. Again, while this was blunted when cells were treated with TNF $\alpha$, siRNAmediated knockdown of Fyn prevented this chronic TNF $\alpha$ inhibition of metformin-stimulated autophagy activation (Figure 8D, 8E and 8F).

To confirm these results, we utilized the GFPLC3 puncta formation assay. As shown Figure 9A, 9B and supplementary Figure 3A, AICAR increased the number of puncta in each cell, which is consistent with an increase in autophagic activity. While TNF $\alpha$ suppressed the AICAR induction of GFP-LC3 puncta, this was essentially completely reversed by siRNA-mediated knockdown of Fyn (Figure 9A, 9B and supplementary Figure 3A). While GFP-LC3 puncta formation assay indicates the levels of LC3 lipidation, it is not always correlated with the increase of autophagy flow. To confirm our results, we utilized the lysosomotrophic agent Bafilomycin A1 to inhibit the autophagy flow. As shown Figure 9C, 9D and supplementary Figure 3C and 3D, Bafilomycin A1 treatment significantly increased GFP-LC3 puncta formation in the basal state and AICAR accelerated this process, indicating that even in basal state the HEK293T cells have a certain level of autophagy flow that is increased by AICAR. Importantly Bafilomycin A1 was unable to increase GFP-LC3 puncta in the absence or presence of AICAR in TNF $\alpha$-treated cells. In contrast, siRNA-mediated knockdown of Fyn restored the autophagy flow in the TNF $\alpha$-treated cells both in the absence and presence of AICAR (Figure 9C, 9D and supplementary Figure 3B and 3C).

Since prolonged exposure of inflammatory mediators inhibit autophagy resulting in severe cell damage and apoptosis [10], we next examined the role of Fyn in apoptosis. As shown Figure 10A and 10B, AICAR did not change the basal state level of apoptosis reflected by PARP cleavage. This lack of AICAR protection is likely due to a limited basal apoptotic response in HEK293T cells, a fact that we confirmed as we were unable to detect CHOP induction in these cells (not shown). However under these conditions, TNF $\alpha$ slightly induced PARP cleavage, which was rescued by AICAR, indicating that TNF $\alpha$-induced apoptosis could be regulated by AMPK. Interestingly siRNA-mediated knockdown of Fyn resulted in a complete loss of PARP cleavage indicating that apoptosis was completely blocked (Figure 10A and 10B). These data indicate that $\mathrm{TNF} \alpha$-induced apoptosis might partially be regulated by AMPK through Fyn, although our data also suggest that Fyn regulates apoptosis independently of AMPK.

\section{DISCUSSION}

AMPK is a master energy sensor in cells and it is activated in various conditions where cellular ATP is decreased. AMPK also has a major role in pathological conditions (e.g. metabolic syndrome, cancer, Alzheimer's disease) and therapeutic activation of AMPK is believed to have beneficial advantages for several disease processes. For example, metformin increases AMPK activity and is the most commonly prescribed oral anti-diabetic agent $[34,35]$ and recent studies have also shown that metformin therapy decreases cancer risk [37].

AMPK is a heterotrimeric complex composed of three different subunits $\alpha, \beta, \gamma$ and currently only two AMPK kinases (LKB1 and CaMKK2) and two AMPK phosphatases (PP2A and $\mathrm{PP} 2 \mathrm{C}$ ) are known to regulate AMPK activity $[17,18,38,39]$. AMPK regulation is mainly affected by the tissue distribution of these upstream kinases. For example, LKB1 is expressed in peripheral tissues (liver, skeletal muscle and adipose) while CaMKK2 is present in neuronal cells [40, 41]. We identified one member of the Src family of proto-oncogene non-receptor tyrosine kinases, Fyn that regulates AMPK activity in peripheral tissues by directly interacting with LKB1 [21].

In this study, we now demonstrate that Fyn also directly regulates AMPK. We found that Fyn phosphorylates the AMPK $\alpha$ subunit on Y436 and this phosphorylation suppresses AMPK activity. The AMPK $\alpha$ subunit is composed of a kinase domain (KD) and a regulatory $\mathrm{C}$-terminus. The regulatory $\mathrm{C}$-terminus has an auto-inhibitory domain (AID), a sensor ( $\alpha$ RIM) loop and a $\beta$-subunit interaction domain ( $\beta$-ID). Additionally, a recent publication has implicated that AMP binding to CBS-3 of $\gamma$ subunit induces the interaction of AID- $\alpha$ RIM2 and $\gamma$ unit resulting in AMPK activation by releasing AID inhibition of the kinase domain [42]. Interestingly, inspection of AMPK sequence revealed that Y436 is localized in $\beta$-ID immediately after the $\alpha$ RIM- 2 that is $2^{\text {nd }}$ part of $\alpha$ RIM and interacting with AID. This suggested that Y436 phosphorylation could affect AMPK subunits assembly. However, we found that Y436-dependent inhibition of AMPK activity was not due to the assembly state of the AMPK heterotrimeric complex (Figure 3C, 3D and supplementary Figure 2A, 2B) and since there is no change in the binding affinity between $\alpha$ subunit and $\beta$ subunit by tyrosine phosphorylation of AMPK $\alpha$ subunit, we speculate that phosphorylation of Y436 may modify the AID- $\alpha$ RIM2 interaction. 
A
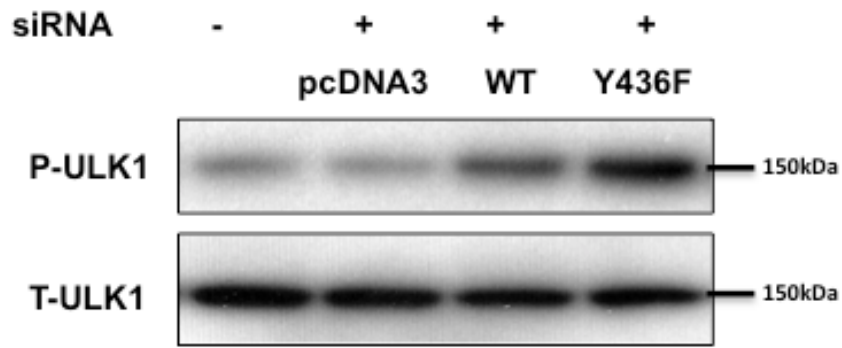

GAPDH

B
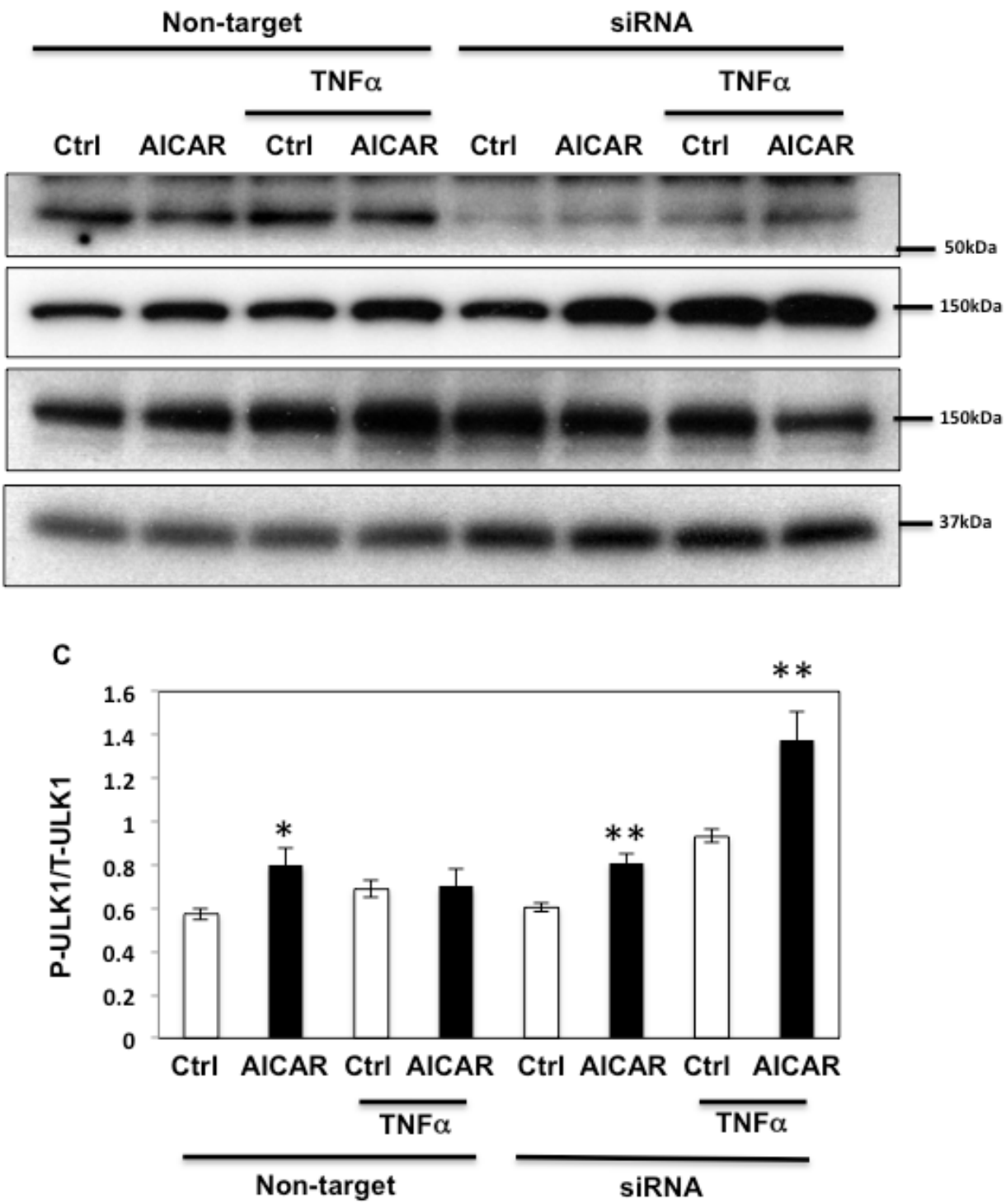

Figure 7: Fyn-mediated AMPK regulation inhibits ULK1 phosphorylation. A. HEK293T cells were co-transfected with siRNAs of human siAMPK $\alpha 1$ and $\alpha 2$ subunits and either empty vector (pcDNA3), GST-rat AMPK $\alpha$ WT (WT) or Y430F mutant (equivalent to human Y436F (Y436F)) to be replaced. Immunoblots were performed $72 \mathrm{~h}$ after transfection. B. HEK293T cells were transfected with Fyn siRNAs. Cells were treated with $10 \mathrm{ng} / \mathrm{ml} \mathrm{TNF} \alpha$ for $36 \mathrm{~h}$ with or without $2 \mathrm{mM}$ AICAR for $10 \mathrm{~min}$. Immunoblots were performed with the indicated antibodies. These are representative images from 3 independent experiments. C. Signal quantifications of the expression levels of phospho-S555 ULK1 to total ULK1. The data are presented as mean \pm SE. Statistical examination between control and AICAR stimulated cells in each sets were performed $* P<0.05,{ }^{*} P<0.01$. 
A

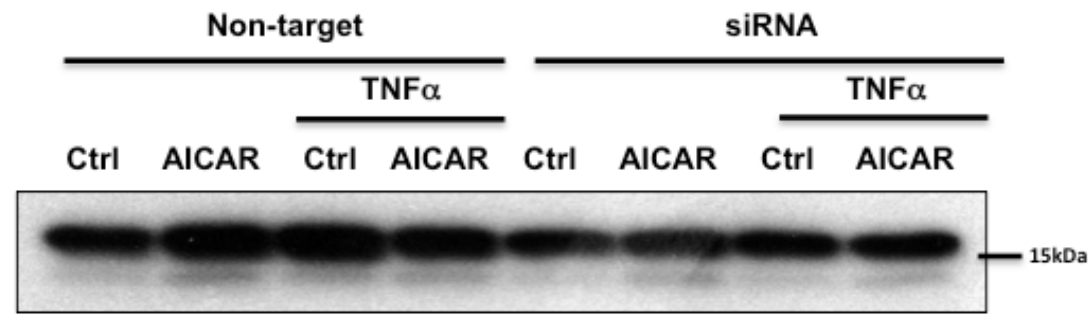

LC3

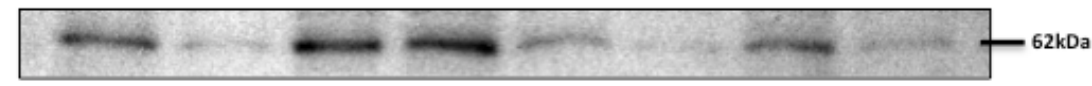

p62

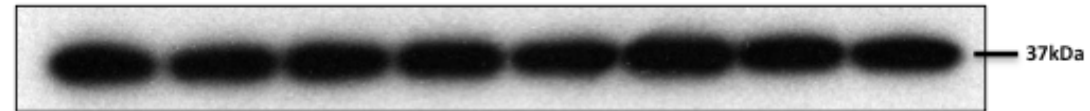

GAPDH

$$
\text { B }
$$

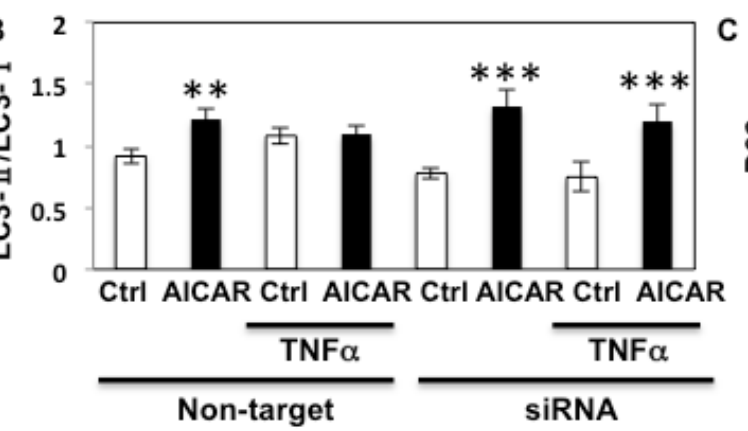

C 3

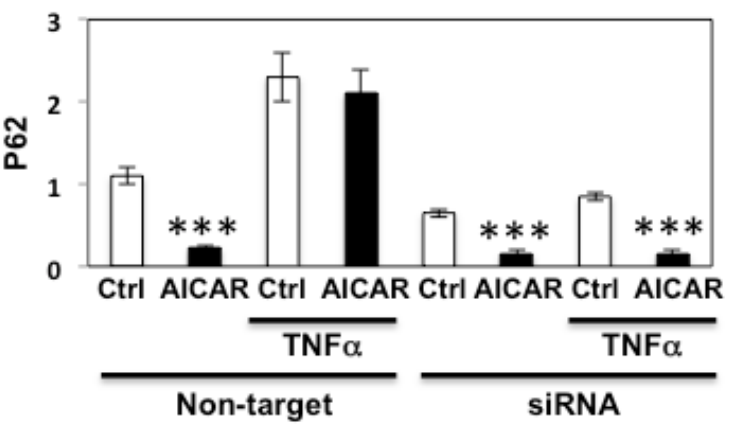

D
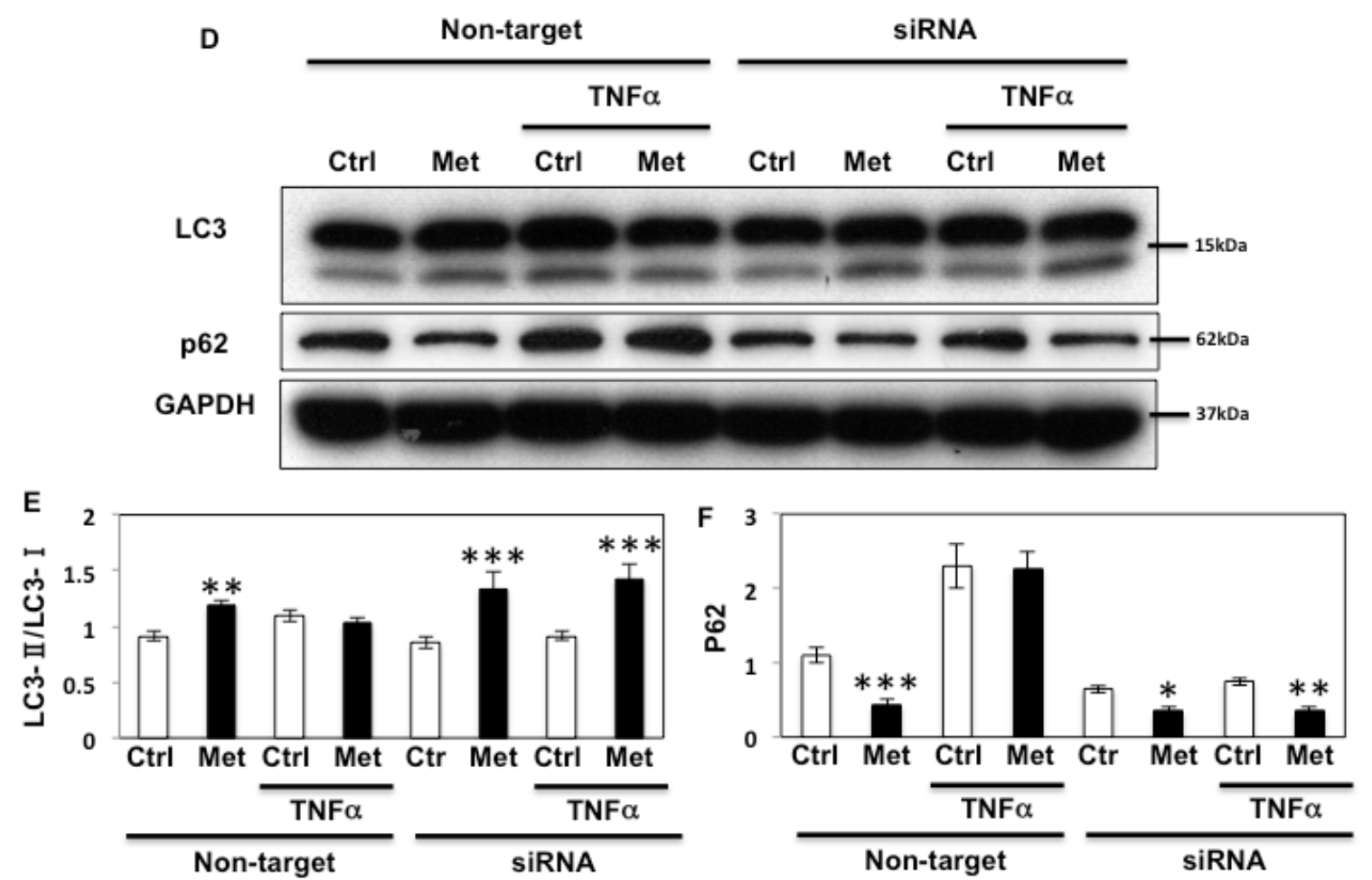

Figure 8: Fyn regulates metabolic stress- induced autophagy through AMPK. A. HEK293T cells were transfected with siRNA for Fyn. The cells were treated with $10 \mathrm{ng} / \mathrm{ml} \mathrm{TNF} \alpha$ for $36 \mathrm{~h}$ with or without $2 \mathrm{mM}$ AICAR for 10 min and immunoblots were performed with the indicated antibodies. These are representative images independently performed 3 times. B., C. Signal quantifications of the ratio of LC3-II to LC3-I (B) and p62/GAPDH (C). The data are presented as mean \pm s.e.m. Statistical examination between control and AICAR stimulated cells in each sets were performed $* * P<0.01, * * * P<0.005$ D. HEK293T cells were transfected with siRNA for Fyn. Cells were treated with $10 \mathrm{ng} / \mathrm{ml} \mathrm{TNF} \alpha$ for $36 \mathrm{~h}$ with or without $5 \mathrm{mM}$ Metformin for $20 \mathrm{~min}$ and immunoblots were performed with the indicated antibodies. These are representative images independently performed 3 times. E., F. Signal quantifications of the ratio of LC3-II to LC3-I (E) and p62/GAPDH (F). The data are presented as mean \pm s.e.m. Statistical examination between control and AICAR stimulated cells in each sets were performed $* * P<0.01, * * * P<0.005$. 
A

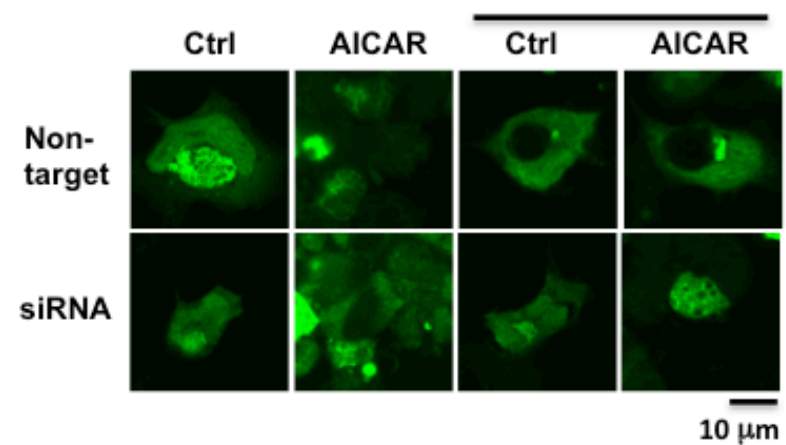

C

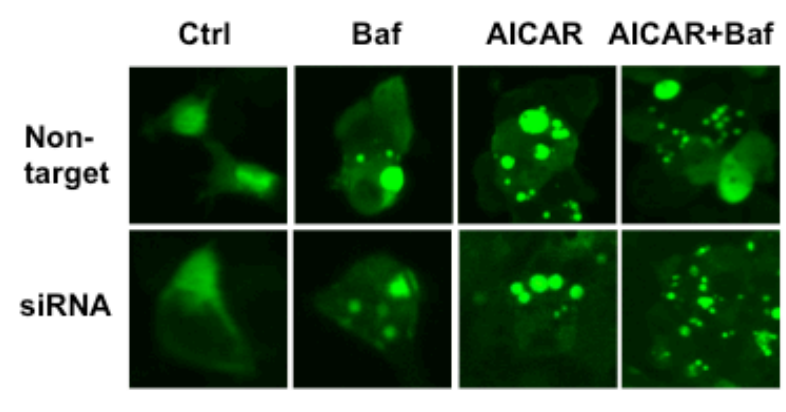

D

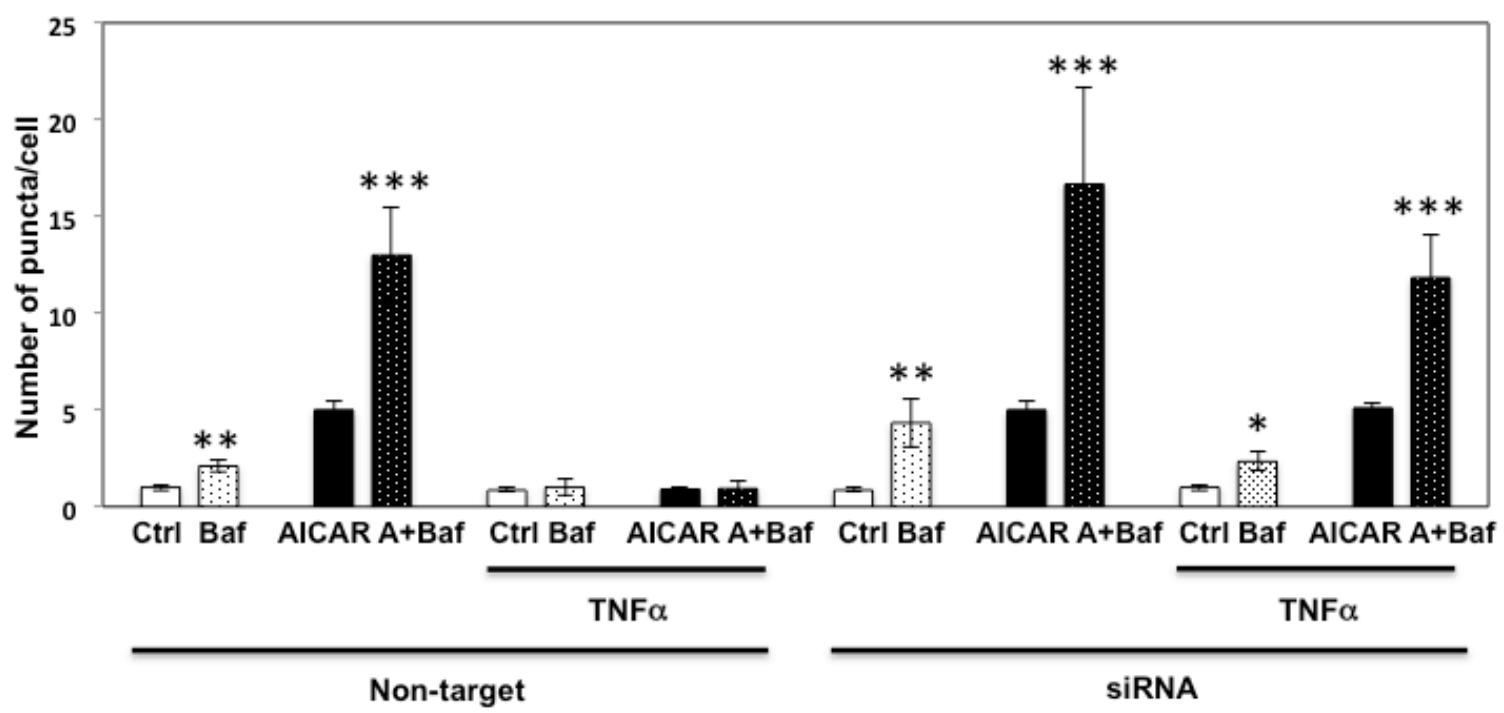

B

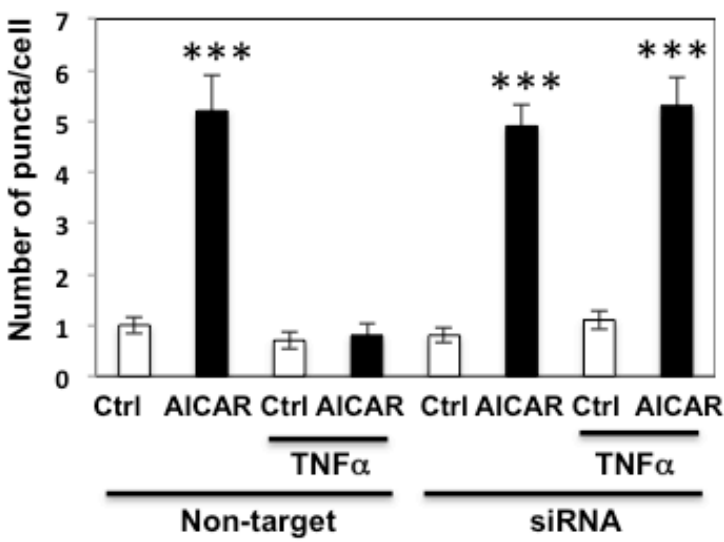

TNF $\alpha$

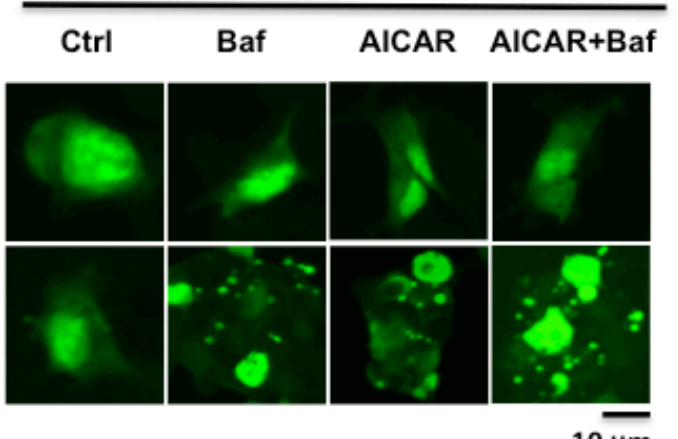

$10 \mu \mathrm{m}$

Figure 9: GFP-LC3 puncta formation assay confirms Fyn's regulation of AICAR dependent autophagy. A. HEK293T cells were transfected with both siRNA for Fyn and GFP-human LC3 construct. The cells were treated with $10 \mathrm{ng} / \mathrm{ml} \mathrm{TNF} \alpha$ for $36 \mathrm{~h}$ with or without $2 \mathrm{mM}$ AICAR for $10 \mathrm{~min}$. Cells were fixed with $4 \%$ paraformaldehyde and mounted with DAKOCytomation Fluorescent Mounting Medium (S3023). These are representative images of high power field from experiments independently performed 3 times. B. Number of puncta in each single cell. Data are representative of $n=5$ experiments. Statistical examination between control and AICARstimulated cells in each sets were performed $* * * P<0.005$. C. HEK293T cells were transfected with both siRNA for Fyn and GFP-human LC3 construct. Cells were treated with $10 \mathrm{ng} / \mathrm{ml} \mathrm{TNF} \alpha$ for $36 \mathrm{~h}$ with or without $100 \mathrm{nM}$ Bafilomycin for $4 \mathrm{~h}$ and $2 \mathrm{mM}$ AICAR for 10 min. Cells were fixed with $4 \%$ paraformaldehyde and mounted with DAKOCytomation Fluorescent Mounting Medium (S3023). These are representative images of high power field from experiments independently performed 3 times. D. Number of puncta in each single cell. Data are representative of $n=5$ experiments. Statistical examination between control and AICAR stimulated cells in each sets were performed $* * * P<0.005$. 
Utilizing TNF $\alpha$, a pro-inflammatory cytokine, we next examined the role of Fyn regulation of AMPK under metabolic stress conditions. Recent studies have reported that $\mathrm{TNF} \alpha$ infusion into lean animals increases PP2C expression, resulting in a reduction in AMPK signaling, fatty acid oxidation, and the development of skeletal muscle insulin resistance [28, 30], but acute incubation of TNF $\alpha$ regulated AMPK activity due to a reduction in the AMP/ATP ratio at least in 3T3L1 adipocytes and in the epithelium-lymphocyte co-culture state $[31,32]$. Therefore we examined the chronic effect of low grade-prolonged TNF $\alpha$ stimulation, which is more likely to reflect in vivo patho-physiological conditions. As expected, prolonged incubation of TNF $\alpha$ suppressed AICAR stimulated T172 $\alpha$ subunit phosphorylation and subsequent ACC phosphorylation, indicating that prolonged TNF $\alpha$ desensitized AMPK activation.
Additionally, we demonstrated that under these conditions Fyn was activated and AMPK was phosphorylated on Y436, suggesting that this desensitization directly results from the Fyn-dependent tyrosine phosphorylation of the AMPK $\alpha$ subunit. Moreover we found that Fyn also regulated AICAR-dependent AMPK activation under prolonged TNF $\alpha$ stimulation.

Recent studies have implicated that AMPK regulates autophagy through a direct AMPK-dependent phosphorylation of ULK1 $[12,13]$ therefore we assessed this as a relevant physiological output. Our data further demonstrate that Fyn-dependent suppression of AMPK activity results in the inhibition of ULK1 phosphorylation and subsequent activation of autophagy, indicated by a induction in the formation of LC3-II, increase in the LC3II/LC-I ratio, decreased p62 expression, GFP-LC3 puncta formation and autophagic flow.

A

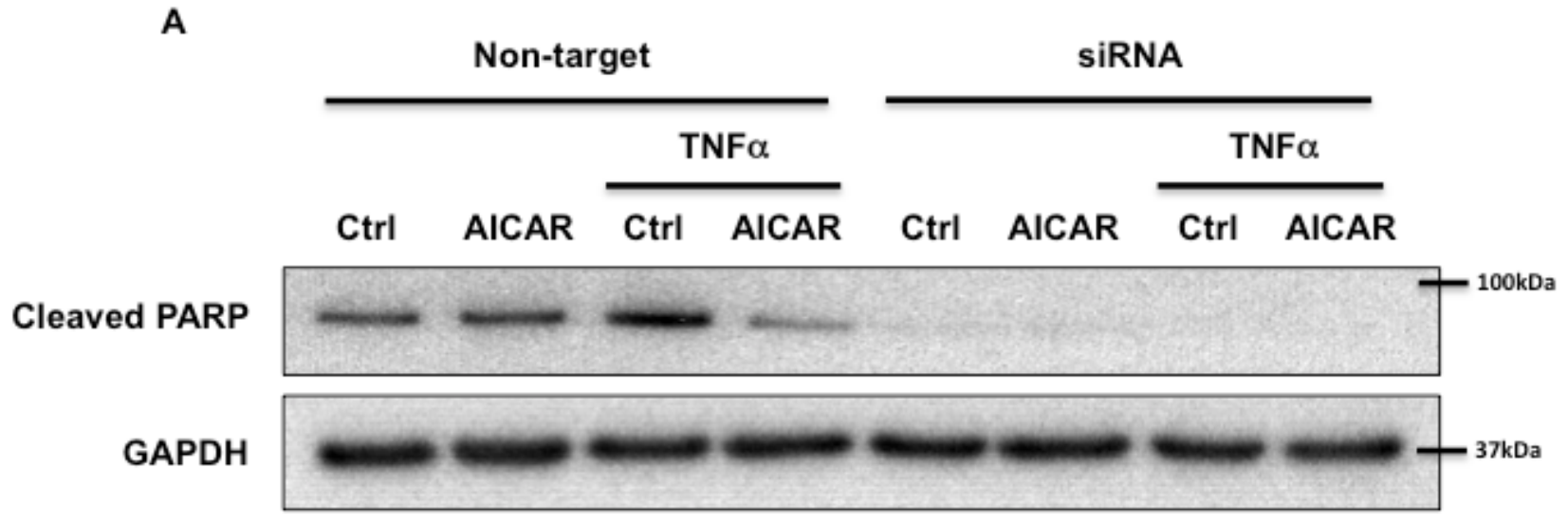

B

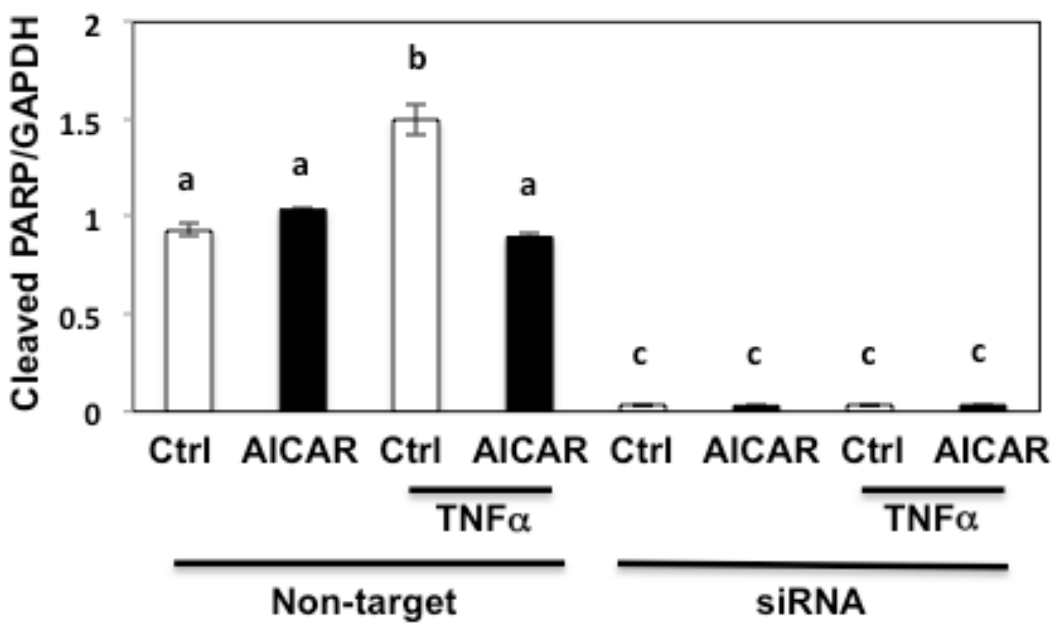

Figure 10: Fyn regulates metabolic stress- induced PARP cleavage through AMPK. A. HEK293T cells were transfected with siRNA for Fyn and treated with $10 \mathrm{ng} / \mathrm{ml} \mathrm{TNF} \alpha$ for $36 \mathrm{~h}$ with or without $2 \mathrm{mM}$ AICAR for $10 \mathrm{~min} .150 \mu \mathrm{g}$ of Lysate were immunoblotted by cleaved PARP pAb (\#5625, Cell Signaling) and GAPDH mAb. These are representative images from experiments independently performed 3 times. B. Signal quantifications of cleaved PARP/GAPDH. The data are presented as mean \pm s.e.m. Non-identical letters (a, b and c) indicate results that are statistically different from each other at $p<0.05$. 
Since autophagy induction by AMPK activation rescues cells from cell death [11], we examined PARP cleavage and demonstrated that $\mathrm{TNF} \alpha$ induction of apoptosis is inhibited by AICAR. More importantly when Fyn was knockdown, apoptosis was completely blocked indicating that AMPK regulates TNF $\alpha$-induced apoptosis and that Fyn plays a significant role in this process. However, since autophagic activity was not affected in Fyn knockdown cells and apoptosis was not induced suggest the presence of alternative pathways autophagy regulating apoptosis by Fyn.

In summary, we found that Fyn directly phosphorylates the $\alpha$ subunit of AMPK on Y436 and this phosphorylation suppresses AMPK activity, subsequently resulting in increased ACC and ULK1 phosphorylation. Prolonged exposure to pro-inflammatory cytokines such as TNF $\alpha$ decreased AICAR-dependent AMPK activation through activation of Fyn. These modulatory events manifest in the inhibition of AMPK-dependent autophagy activation and subsequent apoptosis, suggesting that targeting Fyn activity may have positive therapeutic potential as a novel pathway to activate AMPK.

\section{EXPERIMENTAL PROCEDURES}

\section{Antibodies and reagents}

Flag antibody was from Sigma-Aldrich (St. Louis, MO, USA), the 4G10 and GST antibodies were from Millipore (Bilerica, MA, USA), GAPDH and V5 antibodies were purchased from MBL (Woods Hole, MA, USA), Fyn antibody was from Santa Cruz Biotechnology (Santa Cruz, CA, USA), LC3 antibody was from Novus Biologicals (Littleton, CO, USA) and p62 antibody was from Enzo Life sciences (Exeter, UK). All other antibodies were purchased from Cell Signaling (Boston, MA, USA). Bafilomycin A1 and AICAR were from Sigma-Aldrich (St. Louis, MO, USA). TNF $\alpha$ and Metformin were from EMD Millipore (Bilerica, MA, USA), Toronto Research Chemicals (Toronto, Ontario, Canada) and Cayman Chemical (Ann Arbor, MI, USA) respectively. Leupeptin Hemisulfate was from Fisher Scientific (Pittsburgh, PA, USA). DharmaFECT Duo Transfection Reagent and siRNAs for AMPK $\alpha 1, \alpha 2$ and Fyn were purchased from GE Healthcare (Little Chalfont, UK). X-tremeGENE HP DNA Transfection Reagent were from Roche (Basel, Switzerland). All other reagents were purchased from Sigma-Aldrich.

\section{cDNA constructs}

The pCMV6 Entry Myc-DDK tagged ORF clone of Homo sapiens AMPK $\alpha 2$ construct was obtained from Origene (Rockville, MD, USA) and used to generate the pCMV6-3Myc-2-AMPKa2Myc DDK construct. The gene encoding AMPK $\alpha 2$ was amplified with the oligonucleotides: 5'- $\quad$ GAATTCGCCCGGGCGGGATCCCCCG GGCTGCAGATGGCTGAGAAGCAGAAGCA-3' and 5'- AAGCTTGGTACCGGGCCCCCCCTCGAGGTCGA CGGTATCGATAACCTTATCGTCGTCATCCT-3'

and cloned to pCR-BluntII-TOPO (Invitrogen, CA, USA). This construct and pCMV-3Myc-2 (Agilent Technologies, Santa Clara, CA, USA) were digested with EcoRI and HindIII to obtain pCMV-3Myc-2-AMPKa2Myc-DDK. GST-AMPKa1(r), pcDNA3, pcDNA3Fyn-CA, pcDNA3-LKB1 and eGFP-hLC3 construct were constructed or obtained before [21]. Human Y436 of AMPK $\alpha 2$ subunit is equivalent to rat Y430 of AMPK $\alpha 1$. Y430F of GST-AMPK $\alpha 1$ was obtained by QuickChange 2-XL Site-Directed Mutagenesis Kit (Agilent Technologies, Santa Clara, CA, USA) with the pair of oligonucleotides: 5'- GGAAGGT TGTAAACCCCTTTTATTTGCGTGTGCGAAG-3 and 5'- CTTCGCAC ACGCAAATAAAAGGGGTTTACAACCTTCC-3’

\section{Animals}

Mice over expressing the murine FynB and FynT cDNA were established as previously described [27]. pp5 $9^{f y n}$ knockout mice and their controls were obtained from The Jackson Laboratory (Bar Harbor, ME, USA). Animals were housed in a facility equipped with a $12 \mathrm{~h}$ light/dark cycle and fed ad libitum a standard chow diet (Research Diets, New Brunswick, NJ) containing 67\% (Kcal) carbohydrates, $19 \%$ protein, and $4 \%$ fat. All studies were approved by and performed in compliance with the guidelines of the Albert Einstein College of Medicine Institutional Animal Care and Use Committee (IACUC).

\section{Cell culture}

HEK293T, NIH3T3 and HeLa cells (ATCC, Manassas, VA, USA) were cultured in Dulbecco's Modified Eagle's Medium (Invitrogen, CA, USA) with $10 \%$ Fetal Bovine Serum (Atlanta Biologicals, Flowery Branch, GA, USA) and 1\% Penicillin-Streptomycin (Life technologies, Norwalk, CT, USA). Cells were incubated at $37^{\circ} \mathrm{C}$ moisturized incubator with $5 \% \mathrm{CO}_{2}$. DNA Transfection was performed using X-tremeGENE HP DNA Transfection Reagent and siRNA transfection was performed using DharmaFECT Duo Transfection Reagent according to the manufacture's instructions. siGENOME Human AMPK $\alpha 1$ and $\alpha 2$ and FYN siRNA SMART pool and Non-target siRNA were from GE Healthcare. We saw the effect of AICAR mediating AMPK phosphorylation and activity was fully regulated under confluent condition in HEK293T cells, so every experiments with HEK293T 
cells were performed under confluent cells condition.

\section{Western blot analysis}

Tissues and cell extracts were prepared as described before [27]. Briefly, homogenates were centrifuged for 30 min at $14,000 \mathrm{rpm}$ at $4^{\circ} \mathrm{C}$, and supernatants were collected. Protein concentration was determined with the $\mathrm{BCA}^{\mathrm{TM}}$ Protein Assay (Thermo Scientific, Rockford, IL). Protein lysates $(40 \mu \mathrm{g})$ were separated on 8 to $15 \%$ reducing polyacrylamide gels and transferred onto Immobilon-P polyvinylidene difluoride membranes. Immunoblots were blocked with $5 \%$ milk in Tris-buffered saline or Odyssey Bocking Buffer (Li-COR Biotechnology, Lincoln, NE) for $60 \mathrm{~min}$ at room temperature and incubated overnight at $4^{\circ} \mathrm{C}$ with the indicated antibodies in Tris-buffered saline and $0.05 \%$ Tween 20 (TBST) containing 1\% BSA. Blots were washed in TBST and incubated with horseradish peroxidase-conjugated secondary antibodies $(1: 30,000)$ for $30 \mathrm{~min}$ at room temperature. Membranes were washed in TBST, and antigen-antibody complexes were visualized by chemiluminescence using an ECL kit (Pierce). Alternatively, immunoblots were incubated with IRDye $800 \mathrm{CW}$ Goat Anti Mouse $(\mathrm{H}+\mathrm{L})$ or IRDye680 Goat Anti Rabbit $(\mathrm{H}+\mathrm{L})$ secondary antibodies and signal was detected with the Odyssey Infrared Imaging System (LiCOR Biotechnology).

\section{Immunoprecipitation}

Protein from cells and tissues were prepared in a buffer containing 50mM Tris ( $\mathrm{pH} 7.4), 1 \%$ glycerol and $1 \%$ NP40 supplemented with protease inhibitor (Complete mini, Roche Pharmaceuticals, Nutley, NJ). Homogenates were centrifuged for $30 \mathrm{~min}$ at $14,000 \mathrm{rpm}$ at $4^{\circ} \mathrm{C}$, and supernatants were collected. Protein concentration was determined with the $\mathrm{BCA}^{\mathrm{TM}}$ Protein Assay. Lysates (5-6 $\mathrm{mg}$ ) were immunoprecipitated with indicated antibodies followed by incubation with ProteinA/G plus. Samples were washed 3 times and boiled and separated onto 10\% SDS-PAGE followed by an overnight transfer. Western blot analysis was performed with the indicated antibodies. Alternatively, immunoprecipitation was performed with Catch and Release ${ }^{\circledR}$ v2.0 Reversible Immunoprecipitation System (EMD Millipore) according to manufacturer's protocol, followed by immunoblotting with the indicated antibodies.

\section{In vitro AMPK phosphorylation assay}

AMPK $\alpha 2$ subunit fusion protein and FynT was purchased from Abnova and BPS bioscience, respectively. AMPK $\alpha 2$ protein $(1 \mu \mathrm{g})$ was incubated with the recombinant FynT kinase active $(1.8 \mathrm{U})$ in presence of ATP and kinase buffer (Cell Signaling) and kinase reaction was performed for $1 \mathrm{~h}$ at $35^{\circ} \mathrm{C}$. Samples were separated on 10\% SDS-polyacrylamide gels and immunoblotting was performed with 4G10 monoclonal antibody.

\section{Fyn activity assay}

Fyn activity assay was performed with Universal Tyrosine Kinase Assay kit (Takara Bio Inc, Shiga, Japan) as described previously [27]. Briefly, homogenates were prepared and protein concentration was determined by BCA method. Samples (0.5-1 mg) were incubated with $4 \mu \mathrm{g}$ of Fyn rabbit polyclonal antibody (Santa Cruz Biotechnology) coupled with Catch and Release ${ }^{\circledR}$ v2.0 Reversible Immunoprecipitation System (EMD Millipore). Samples were washed three times with NP40 lysis buffer and once with the kinase reaction buffer. Immuno-complexes were diluted 2.5 fold and $40 \mu \mathrm{l}$ samples and $10 \mu \mathrm{l}$ ATP solution were incubated in an ELISA plate provided. Measurement of the kinase activity was performed according to the manufacturer's protocol.

\section{Immunofuorescence}

HEK293T cells were transfected with GFP-human LC3 construct. Transfected cells were washed with PBS and fixed for $10 \mathrm{~min}$ in PBS containing 4\% PFA. Cells were imaged using a confocal fluorescence microscope (FV10i; Olympus).

\section{Statistics}

All data are presented as mean \pm s.e.m. Significant data were defined as $p<0.05$ using Student's $t$ test or ANOVA.

\section{CONFLICTS OF INTEREST}

The authors do not have any conflicts of interest.

\section{FUNDING}

This work was supported by KAKENHI (25860738).

\section{REFERENCES}

1. Yamada E, Singh R. Mapping autophagy on to your metabolic radar. Diabetes. 2012;61(2):272-80.

2. Altman BJ, Rathmell JC. Metabolic stress in autophagy and cell death pathways. Cold Spring Harbor perspectives in biology. 2012;4(9):a008763. 
3. Singh R, Kaushik S, Wang Y, Xiang Y, Novak I, Komatsu M, Tanaka K, Cuervo AM, Czaja MJ. Autophagy regulates lipid metabolism. Nature. 2009;458(7242):1131-5.

4. Auranen M, Villanova M, Muntoni F, Fardeau M, Scherer SW, Kalino H, Minassian BA. X-linked vacuolar myopathies: two separate loci and refined genetic mapping. Annals of neurology. 2000;47(5):666-9.

5. Raben N, Schreiner C, Baum R, Takikita S, Xu S, Xie T, Myerowitz R, Komatsu M, Van der Meulen JH, Nagaraju K, Ralston E, Plotz PH. Suppression of autophagy permits successful enzyme replacement therapy in a lysosomal storage disorder--murine Pompe disease. Autophagy. 2010;6(8):1078-89.

6. Singh R, Xiang Y, Wang Y, Baikati K, Cuervo AM, Luu YK, Tang Y, Pessin JE, Schwartz GJ, Czaja MJ. Autophagy regulates adipose mass and differentiation in mice. The Journal of clinical investigation. 2009;119(11):3329-39.

7. Zhang Y, Goldman S, Baerga R, Zhao Y, Komatsu M, Jin S. Adipose-specific deletion of autophagy-related gene 7 (atg7) in mice reveals a role in adipogenesis. Proceedings of the National Academy of Sciences of the United States of America. 2009;106(47):19860-5.

8. Ebato C, Uchida T, Arakawa M, Komatsu M, Ueno T, Komiya K, Azuma K, Hirose T, Tanaka K, Kominami E, Kawamori R, Fujitani Y, Watada H. Autophagy is important in islet homeostasis and compensatory increase of beta cell mass in response to high-fat diet. Cell metabolism. 2008;8(4):325-32.

9. Jung HS, Chung KW, Won Kim J, Kim J, Komatsu M, Tanaka K, Nguyen YH, Kang TM, Yoon KH, Kim JW, Jeong YT, Han MS, Lee MK, et al. Loss of autophagy diminishes pancreatic beta cell mass and function with resultant hyperglycemia. Cell metabolism. 2008;8(4):31824.

10. Lapaquette P, Guzzo J, Bretillon L, Bringer MA. Cellular and Molecular Connections between Autophagy and Inflammation. Mediators of inflammation. 2015;2015:398483.

11. Law BY, Mok SW, Chan WK, Xu SW, Wu AG, Yao XJ, Wang JR, Liu L, Wong VK. Hernandezine, a novel AMPK activator induces autophagic cell death in drug-resistant cancers. Oncotarget. 2016;7:8090-104. doi: 10.18632/ oncotarget.6980.

12. Kim J, Kundu M, Viollet B, Guan KL. AMPK and mTOR regulate autophagy through direct phosphorylation of Ulk1. Nature cell biology. 2011;13(2):132-41.

13. Egan DF, Shackelford DB, Mihaylova MM, Gelino S, Kohnz RA, Mair W, Vasquez DS, Joshi A, Gwinn DM, Taylor R, Asara JM, Fitzpatrick J, Dillin A, et al. Phosphorylation of ULK1 (hATG1) by AMP-activated protein kinase connects energy sensing to mitophagy. Science. 2011;331(6016):456-61.

14. Crute BE, Seefeld K, Gamble J, Kemp BE, Witters LA. Functional domains of the alphal catalytic subunit of the
AMP-activated protein kinase. The Journal of biological chemistry. 1998;273(52):35347-54.

15. Hardie DG, Ross FA, Hawley SA. AMPK: a nutrient and energy sensor that maintains energy homeostasis. Nature reviews Molecular cell biology. 2012;13(4):251-62.

16. Woods A, Cheung PC, Smith FC, Davison MD, Scott J, Beri RK, Carling D. Characterization of AMP-activated protein kinase beta and gamma subunits. Assembly of the heterotrimeric complex in vitro. The Journal of biological chemistry. 1996;271(17):10282-90.

17. Hawley SA, Pan DA, Mustard KJ, Ross L, Bain J, Edelman AM, Frenguelli BG, Hardie DG. Calmodulin-dependent protein kinase kinase-beta is an alternative upstream kinase for AMP-activated protein kinase. Cell metabolism. 2005;2(1):9-19.

18. Woods A, Johnstone SR, Dickerson K, Leiper FC, Fryer LG, Neumann D, Schlattner U, Wallimann T, Carlson $\mathrm{M}$, Carling D. LKB1 is the upstream kinase in the AMPactivated protein kinase cascade. Current biology : CB. 2003;13(22):2004-8.

19. Kajita K, Mune T, Ikeda T, Matsumoto M, Uno Y, Sugiyama C, Matsubara K, Morita H, Takemura M, Seishima M, Takeda J, Ishizuka T. Effect of fasting on PPARgamma and AMPK activity in adipocytes. Diabetes research and clinical practice. 2008;81(2):144-9.

20. Bastie CC, Zong H, Xu J, Busa B, Judex S, Kurland IJ, Pessin JE. Integrative metabolic regulation of peripheral tissue fatty acid oxidation by the SRC kinase family member Fyn. Cell metabolism. 2007;5(5):371-81.

21. Yamada E, Pessin JE, Kurland IJ, Schwartz GJ, Bastie CC. Fyn-dependent regulation of energy expenditure and body weight is mediated by tyrosine phosphorylation of LKB1. Cell metabolism. 2010;11(2):113-24.

22. Zhang S, Qi Q, Chan CB, Zhou W, Chen J, Luo HR, Appin C, Brat DJ, Ye K. Fyn-phosphorylated PIKE-A binds and inhibits AMPK signaling, blocking its tumor suppressive activity. Cell death and differentiation. 2016;23(1):52-63.

23. Salmond RJ, Filby A, Qureshi I, Caserta S, Zamoyska R. T-cell receptor proximal signaling via the Src-family kinases, Lck and Fyn, influences T-cell activation, differentiation, and tolerance. Immunological reviews. 2009;228(1):9-22.

24. Palacios EH, Weiss A. Function of the Src-family kinases, Lck and Fyn, in T-cell development and activation. Oncogene. 2004;23(48):7990-8000.

25. Saito YD, Jensen AR, Salgia R, Posadas EM. Fyn: a novel molecular target in cancer. Cancer. 2010;116(7):1629-37.

26. Davidson D, Viallet J, Veillette A. Unique catalytic properties dictate the enhanced function of p59fynT, the hemopoietic cell-specific isoform of the Fyn tyrosine protein kinase, in T cells. Molecular and cellular biology. 1994;14(7):4554-64.

27. Yamada E, Bastie CC, Koga H, Wang Y, Cuervo AM, Pessin JE. Mouse skeletal muscle fiber-type-specific 
macroautophagy and muscle wasting are regulated by a Fyn/STAT3/Vps34 signaling pathway. Cell reports. 2012;1(5):557-69.

28. Steinberg GR, Kemp BE. AMPK in Health and Disease. Physiological reviews. 2009;89(3):1025-78.

29. Harris J. Autophagy and cytokines. Cytokine. 2011;56(2):140-4.

30. Steinberg GR, Michell BJ, van Denderen BJ, Watt MJ, Carey AL, Fam BC, Andrikopoulos S, Proietto J, Görgün CZ, Carling D, Hotamisligil GS, Febbraio MA, Kay TW, et al. Tumor necrosis factor alpha-induced skeletal muscle insulin resistance involves suppression of AMP-kinase signaling. Cell metabolism. 2006;4(6):465-74.

31. Tang XX, Chen H, Yu S, Zhang L, Caplan MJ, Chan HC. Lymphocytes accelerate epithelial tight junction assembly: role of AMP-activated protein kinase (AMPK). PloS one. 2010;5(8):e12343.

32. Hong SW, Lee J, Park SE, Rhee EJ, Park CY, Oh KW, Park SW, Lee WY. Activation of AMP-Activated Protein Kinase Attenuates Tumor Necrosis Factor-alpha-Induced Lipolysis via Protection of Perilipin in 3T3-L1 Adipocytes. Endocrinol Metab (Seoul). 2014;29(4):553-60.

33. Bollheimer LC, Buettner R, Pongratz G, Brunner-Ploss R, Hechtl C, Banas M, Singler K, Hamer OW, Stroszczynski C, Sieber CC, Fellner C. Sarcopenia in the aging high-fat fed rat: a pilot study for modeling sarcopenic obesity in rodents. Biogerontology. 2012;13(6):609-20.

34. Corton JM, Gillespie JG, Hawley SA, Hardie DG. 5-aminoimidazole-4-carboxamide ribonucleoside. A specific method for activating AMP-activated protein kinase in intact cells? European journal of biochemistry / FEBS. 1995;229(2):558-65.

35. Grahame Hardie D. AMP-activated protein kinase: a key regulator of energy balance with many roles in human disease. Journal of internal medicine. 2014;276(6):543-59.
36. Mihaylova MM, Shaw RJ. The AMPK signalling pathway coordinates cell growth, autophagy and metabolism. Nature cell biology. 2011;13(9):1016-23.

37. Snima KS, Pillai P, Cherian AM, Nair SV, Lakshmanan VK. Anti-diabetic drug metformin: challenges and perspectives for cancer therapy. Current cancer drug targets. 2014;14(8):727-36

38. Davies SP, Helps NR, Cohen PT, Hardie DG. 5'AMP inhibits dephosphorylation, as well as promoting phosphorylation, of the AMP-activated protein kinase. Studies using bacterially expressed human protein phosphatase-2C alpha and native bovine protein phosphatase-2AC. FEBS letters. 1995;377(3):421-5.

39. Marley AE, Sullivan JE, Carling D, Abbott WM, Smith GJ, Taylor IW, Carey F, Beri RK. Biochemical characterization and deletion analysis of recombinant human protein phosphatase 2C alpha. The Biochemical journal. 1996;320 ( Pt 3):801-6.

40. Jensen TE, Rose AJ, Jørgensen SB, Brandt N, Schjerling P, Wojtaszewski JF, Richter EA. Possible CaMKK-dependent regulation of AMPK phosphorylation and glucose uptake at the onset of mild tetanic skeletal muscle contraction. American journal of physiology Endocrinology and metabolism. 2007;292(5):E1308-17.

41. Anderson KA, Means RL, Huang QH, Kemp BE, Goldstein EG, Selbert MA, Edelman AM, Fremeau RT, Means AR. Components of a calmodulin-dependent protein kinase cascade. Molecular cloning, functional characterization and cellular localization of $\mathrm{Ca} 2+/$ calmodulin-dependent protein kinase kinase beta. The Journal of biological chemistry. 1998;273(48):31880-9.

42. Li X, Wang L, Zhou XE, Ke J, de Waal PW, Gu X, Tan MH, Wang D, Wu D, Xu HE, Melcher K. Structural basis of AMPK regulation by adenine nucleotides and glycogen. Cell research. 2015;25(1):50-66. 